\title{
Fuzzy preorders: conditional extensions, extensions and their representations
}

\author{
J. C. R. Alcantud \\ University of Salamanca \\ http://borda.usal.es \\ jcr@usal.es
}

\author{
S. Díaz \\ University of Oviedo \\ http://unimode.uniovi.es \\ diazsusana@uniovi.es
}

\begin{abstract}
The crisp literature provides characterizations of the preorders that admit a total preorder extension when some pairwise order comparisons are imposed on the extended relation. It is also known that every preorder is the intersection of a collection of total preorders. In this contribution we generalize both approaches to the fuzzy case. We appeal to a construction for deriving the strict preference and the indifference relations from a weak preference relation, that allows to obtain full characterizations in the conditional extension problem. This improves the performance of the construction via generators studied earlier.
\end{abstract}

keyword fuzzy relation, transitivity, acyclicity, consistency, extension, indicator.

\section{Introduction}

This paper studies compatible extensions of fuzzy binary relations. The source of inspiration is the classic Szpilrajn Theorem (cf. [27]), which states that every partial order can be refined to a linear order or put in another form, that any preorder has a total preorder extension (cf., Arrow [3, Chapter VI], Hansson [23, Lemma 3]). This is an important principle that fostered many studies to understand the significance of such refinements. A celebrated application is given by Dushnik and Miller [16], who prove that any partial order is the intersection of linear orders. Relatedly, Donaldson and Weymark [15] (see also Bossert [8]) prove the parallel result for preorders, namely, that any preorder is the intersection of total preorders.

We can also cite various fuzzy versions or extensions of Szpilrajn's theorem, like Georgescu [17, Theorem 5.4] and [18, Corollary 4.37], Gottwald [22, Proposition 2.34] for t-norms withour zero divisors, or Zadeh [30, Theorem 8]. Bodenhofer and Klawonn [5, Theorem 6.7] conduct a detailed investigation of linearity axioms for fuzzy orders. Höhle and Blanchard [25] produce variations for antisymmetric preorders both of the extension theorem in their Theorem 
II.7 and of the intersection theorem in their Corollary II.8. Finally, Alcantud and Díaz [2] provide several new variations of the Szpilrajn theorem for fuzzy preorders, in the spirit of [1]. We emphasize that in order to define the key concept of compatible extension of a fuzzy reflexive relation, [2] relies on the construction of an asymmetric part from the original relation that is based on generators (cf., De Baets and Fodor [11]).

In this paper we provide several new extensions of Szpilrajn's theorem for fuzzy relations.

Firstly we depart from the precedent [2] in order to consider the alternative approach to the construction of an asymmetric part from the original preference relation in Georgescu [17]. We prove further results in the tradition of [1] (also $[2])$, namely, on the identification of constraints that can be imposed to the fuzzy total preorder extensions.

Secondly, we put forward new results inspired by the alternative approaches by [15] and [25]. Hence we explore the problem of extending fuzzy relations with fixed degrees of relation between pairs of elements. Then this technique is applied to provide some general representation results for fuzzy relations. But in addition to these novelties, along such programme we introduce indicators into the analysis. We are interested in indicators which capture the degree to which a fuzzy relation verifies a given property (e.g., reflexivity or min-transitivity). The recourse to this notion permits to state properties of fuzzy relations which are especially appealing when they are fuzzy preorders. Put differently, we demonstrate that also in our framework (e.g., the investigation of representation theorems), indicators allow us to extend statements on fuzzy preorders to fuzzy relations.

This paper is organized as follows. In Section 2 we recall the results by Alcantud [1] that inspire this paper, as generalized in [2]. In Section 3 we give some notation and preliminaries on fuzzy relations and their extensions. Section 4 solves our main problems about conditional fuzzy extensions.

Section 5 introduces the approach by indicators in the context of [25]. Then we take advantage of these results to prove our representation theorem of a fuzzy preorder as the intersection of its fuzzy total preorder extensions, also in terms of indicators for unrestricted fuzzy relations. Section 6 contains some concluding remarks.

\section{Crisp conditional extensions}

In the context of crisp or classical relations, Alcantud [1] proves some characterizations of preorders for which a total preorder exists, satisfying some additional conditions as its extension. According to Alcantud and Díaz [2] those characterizations can be sligthtly extended within the crisp context. In this section we recall the main results concerning classical relations in [2], including basic definitions in crisp set theory.

Definition 2.1. A binary relation $Q$ on a universe $X$ is reflexive if a $Q$ a for all $a \in X$. A weak preference is a reflexive relation. 
Associated with any weak preference relation $R$ we can build its symmetric component, called indifference relation and denoted by $I_{R}$ or simply $I$ when there is no danger of confusion. It is defined as $a I_{R} b$ if and only if $a R b \wedge b R a$. We can also obtain an asymmetric component, called strict preference relation and denoted by $P_{R}$ or simply $P$ when there is no danger of confusion. It is defined as $a P b$ if and only if $a R b \wedge \neg b R a$.

Weak preference relations $R$ are usually understood as follows: if $a$ is considered at least as good as $b$ then $a R b$, otherwise $\neg a R b$.

Definition 2.2. A binary relation $Q$ on a universe $X$ is

(a) transitive if for all $a, b, c \in X$ it holds that ( $a Q b$ and $b Q c)$ imply $a Q c$,

(b) acyclic if for any $a_{1}, \ldots, a_{n} \in X$ it holds that

$$
\left(a_{1} Q a_{2} \text { and } a_{2} Q a_{3} \text { and } \ldots \text { and } a_{n-1} Q a_{n}\right) \quad \text { imply } \neg a_{n} Q a_{1} \text {, }
$$

(c) transitive-consistent if it is a weak preference relation $R$ with strict preference relation $P$ such that for any $a_{1}, \ldots, a_{n} \in X$ it holds that

$$
\left(a_{1} P a_{2} \text { and } a_{2} R a_{3} \text { and } \ldots \text { and } a_{n-1} R a_{n}\right) \quad \text { imply } \neg a_{n} R a_{1} \text {, }
$$

(d) total if $a Q b$ or $b Q a$ for all $a \neq b \in X$,

(e) complete if $a Q b$ or $b Q a$ for all $a, b \in X$,

(f) a preorder if it is transitive and reflexive,

Clearly, transitive-consistency is a condition weaker than transitivity.

Definition 2.3. The transitive closure of a relation $Q$ is the smallest transitive relation $Q^{T}$ such that $Q \subseteq Q^{T}$.

It is easy to prove that the transitive closure of a relation $Q$ is the intersection of all the transitive relations that contain the relation $Q$.

Definition 2.4. If $Q \subseteq Q^{\prime}$, then $Q^{\prime}$ is an extension of $Q$. Given a weak preference relation $R_{1}$, a compatible extension of $R_{1}$ is another weak preference relation $R_{2}$ defined on the same universe, such that $R_{1} \subseteq R_{2}$ and $P_{R_{1}} \subseteq P_{R_{2}}$, where $P_{R_{i}}$ is the strict preference relation associated with $R_{i}$.

Alcantud [1] provides two characterizations for the class of transitive binary relations that can be extended under certain conditions. His first theorem is stated with the help of the following auxiliary relation:

Definition 2.5. Let $R$ be a binary relation defined on a set of alternatives $X$. Let $X_{I}=\left\{a_{1}, \ldots, a_{n}, b_{1}, \ldots, b_{n}\right\}$ be an ordered set of possibly repeated elements of $X$. The relation $R^{A}$ associated with $X_{I}$ and $R$ is defined by $a_{i} R^{A} a_{j}$ if and only if $a_{i} R b_{j}$. 
Theorem 2.1 (Alcantud [1]). Let $R$ be a preorder on a set $X$. Let $X_{I}=$ $\left\{a_{1}, \ldots, a_{n}, b_{1}, \ldots, b_{n}\right\}$ be an ordered list of possibly repeated elements of $X$. The following statements are equivalent:

a) There is a total preorder $\tilde{R}$ that is a compatible extension of $R$ such that $b_{i} \tilde{P} a_{i}$ for each $i=1, \ldots, n$, where $\tilde{P}$ denotes the asymmetric part of $\tilde{R}$.

b) $R^{A}$ associated with $X_{I}$ and $R$ is acyclic.

In order to recall Alcantud's second theorem we need to introduce new concepts.

Definition 2.6. Let $X_{I}=\left\{a_{1}, \ldots, a_{p}, b_{1}, \ldots, b_{p}\right\}$ be an ordered list of possibly repeated elements of $X$. For each $c \in X_{I}$, let

$$
\delta(c)= \begin{cases}b_{i} & \text { if } c=a_{i}, \\ a_{i} & \text { if } c=b_{i} .\end{cases}
$$

Let $X_{I}^{n}=\left\{a_{n+1}, \ldots, a_{p}, b_{n+1}, \ldots, b_{p}\right\}$. If $n=p$, then $X_{I}^{n}=\varnothing$.

Definition 2.7. The relation $R^{I}$ associated with $R$ and $X_{I}$ is defined as follows:

$$
a R^{I} b \text { if and only if } a R \delta(b) \text {, for all } a, b \in X_{I} .
$$

We say that $R^{I}$ is $\delta$-cyclic along $X_{I}^{n}$ if $c_{1} R^{I} c_{2}$ and $c_{2} R^{I} c_{3}$ and $\ldots c_{k} R^{I} c_{1}$ implies that

$$
\delta\left(c_{1}\right) R^{I} \delta\left(c_{k}\right) \quad \ldots \quad \delta\left(c_{2}\right) R^{I} \delta\left(c_{1}\right), \text { for all } c_{1}, \ldots, c_{k} \in X_{I}^{n} .
$$

Definition 2.8. The relation $R^{G}$ associated with $R, n \leq p$ and $X_{I}$ is defined by

$$
a_{i} R^{G} a_{j}=\left(a_{i} R b_{j} \vee \bigvee_{\left\{c_{1}, \ldots c_{k}\right\} \subseteq X_{I}^{n}}\left(a_{i} R \delta\left(c_{1}\right) \wedge c_{1} R \delta\left(c_{2}\right) \wedge \cdots \wedge c_{k} R b_{j}\right)\right)
$$

Remark 2.1. The relation $R^{G}$ connects those alternatives that will later be connected by the extensions of $R$ that satisfy some additional requirements. That is, if we impose additional connections to the elements in $X_{I}$ and consider a transitive extension $\tilde{R}$ of $R$ satisfying those conditions, the relation $R^{G}$ connects two elements $a_{i} R^{G} a_{j}$ if $a_{i} \tilde{R} b_{j}$.

Definition 2.9. The relation $R^{G}$ is $\delta$-consistent with $X_{I}$ and $n \leq p$ if

$$
a_{i_{1}} R^{G} a_{i_{2}}, \ldots, a_{i_{k}} R^{G} a_{i_{1}} \text { implies } i_{t}>n \text { for all } t \in\{1, \ldots, k\} \text {. }
$$

Theorem 2.2 (Alcantud [1]). Let $R$ be a preorder on a set $X$. Let $X_{I}=$ $\left\{a_{1}, \ldots, a_{p}, b_{1}, \ldots, b_{p}\right\}$ be an ordered list of possibly repeated elements of $X$ and let $n \leq p$. The following statements are equivalent:

a) There exists a total preorder $\tilde{R}$ that is a compatible extension of $R$ such that $b_{i} \tilde{P} a_{i}$ for each $i=1, \ldots, n$, and $b_{i} \tilde{I} a_{i}$ for $i=n+1, \ldots, p$, where $\tilde{P}$ and $\tilde{I}$ denote the asymmetric and symmetric components of $\tilde{R}$ respectively. 
b) The relation $R^{G}$ is $\delta$-consistent with $X_{I}$ and $n$, and $R^{I}$ is $\delta$-cyclic along $X_{I}^{n}$.

Remark 2.2. In Theorem 2.2, the conditions in b) basically request that if the new connections together with the original relations produce cycles, all the elements involved are indifferent. The condition on $R^{G}$ says that if there are cycles among the elements that are going to be connected by the extension, those cycles must involve only elements that are going to be indifferent in the extension. The condition involving the relation $R^{I}$ also prevents the existence of strict preference in cycles: there cannot be strict preferences among elements that are required to be indifferent in the extension. Consider a simple example: take the elements $X_{I}=\left\{a_{1}, a_{2}, a_{3}, b_{1}, b_{2}, b_{3}\right\}$ and assume that the original relation $R$ satisfies $a_{1} R b_{2}, a_{2} R b_{3}$ and $a_{3} R b_{1}$. In order to find an extension such that $a_{i} \tilde{I} b_{i}$ for all $i=1,2,3$, we must have that also $b_{1} R a_{3}, b_{3} R a_{2}$ and $b_{2} R a_{1}$, otherwise, the extension $\tilde{R}$ would have a cycle with at least one strict preference involved.

Both theorems can be extended to transitive-consistent binary relations, in the following terms:

Corollary 2.1 (Alcantud and Díaz [2]). Let $R$ be a (crisp) transitive-consistent weak preference relation on a universe $X$. Let $X_{I}=\left\{a_{1}, \ldots, a_{n}, b_{1}, \ldots, b_{n}\right\}$ be an ordered list of possibly repeated elements of $X$. The following statements are equivalent:

a) There is a total preorder $\tilde{R}$ that is a compatible extension of $R$ such that $b_{i} \tilde{P} a_{i}$ for each $i=1, \ldots, n$, where $\tilde{P}$ denotes the asymmetric component of $\tilde{R}$.

b) $R^{A}$ associated with $X_{I}$ and $R^{T}$ is acyclic.

Corollary 2.2 (Alcantud and Díaz [2]). Let $R$ be a (crisp) transitive-consistent weak preference relation on a universe $X$. Let $X_{I}=\left\{a_{1}, \ldots, a_{p}, b_{1}, \ldots, b_{p}\right\}$ be an ordered list of possibly repeated elements of $X$ and let $n \leq p$. The following statements are equivalent:

a) There exists a total preorder $\tilde{R}$ that is a compatible extension of $R$ such that $b_{i} \tilde{P} a_{i}$ for each $i=1, \ldots, n$ and $b_{i} \tilde{I} a_{i}$ for $i=n+1, \ldots, p$, where $\tilde{P}$ and $\tilde{I}$ denote the asymmetric and symmetric components of $\tilde{R}$ respectively.

b) The relation $R^{G}$ associated with $R^{T}$ is $\delta$-consistent with $X_{I}$ and $n$ and $R^{I}$ (associated with $R^{T}$ ) is $\delta$-cyclic along $X_{I}^{n}$.

\section{$3 \quad$ Fuzzy relations and their extensions}

This section recalls some basic definitions on fuzzy set theory.

Fuzzy set theory was introduced by Zadeh in [29] in order to formalize ambiguous or imprecise reasonings. Contrary to classical logic where statements are true or false, fuzzy theory allows degrees of truth or connection. The set 
$\{0,1\}$ where 0 means false or absence of connection and 1 stands for true or presence of connection, is replaced by the whole interval $[0,1]$. Intermediate values stand for intermediate degrees of truth or connection. The advantage of this theory is the possibility of formalizing human way of thinking in a more accurate way.

Definition 3.1. A fuzzy relation $Q$ defined on a universe $X$ is a mapping $Q: X \times X \rightarrow[0,1]$. For every $a, b \in X, Q(a, b)$ indicates the degree with which $a$ is connected to $b$ by the relation $Q$.

A fuzzy weak preference relation is a reflexive fuzzy relation, i.e., a fuzzy relation $R$ such that $R(a, a)=1$ for all $a \in X$.

Given two fuzzy relations, $Q_{1}$ and $Q_{2}$, we say that $Q_{1}$ is contained in $Q_{2}$, $Q_{1} \subseteq Q_{2}$ if $Q_{1}(a, b) \leq Q_{2}(a, b)$ for all $a, b \in X$.

Now in order to deal with fuzzy relations, we provide the necessary concepts to generalize the classical intersection and negation.

Definition 3.2. A t-norm $T$ is a binary mapping $T:[0,1] \times[0,1] \rightarrow[0,1]$ satisfying the following four properties: commutativity, associativity, monotonicity (in each component) and neutral element 1.

The greatest t-norm is the minimum operator $T_{\mathbf{M}}(x, y)=\min (x, y)$. We also use the common notation $\min (x, y)=x \wedge y$.

A t-norm $T$ has zero divisors if there is a pair of values $(x, y) \in] 0,1\left[{ }^{2}\right.$ such that $T(x, y)=0$. In this case $x$ and $y$ are called zero divisors of $T$. The minimum t-norm does not admit zero divisors.

If we fix one argument, a t-norm can be considered a real function on the other component. If for each component, the corresponding function is left continuous (as a real function), the t-norm is said left continuous.

Definition 3.3. Given a left continuous $t$-norm $T$, its residuum $\rightarrow$ is defined as follows

$$
x \rightarrow y=\sup \{z \in[0,1] \mid T(x, z) \leq y\} .
$$

The negation $\neg$ associated to a left continuous t-norm $T$ is defined as $\neg x=x \rightarrow$ $0=\sup \{y \in[0,1] \mid T(x, y)=0\}$.

The residuum satisfies the following properties:

$$
\begin{aligned}
y \rightarrow z \leq x \rightarrow z & \text { for all } x \leq y, z \in[0,1] . \\
x \leq y \rightarrow x & \text { for all } x, y \in[0,1] . \\
0 \rightarrow x=1 & \text { for all } x \in[0,1] . \\
1 \rightarrow x=x & \text { for all } x \in[0,1] . \\
x \rightarrow y=1 & \text { for all } x \leq y \in[0,1] .
\end{aligned}
$$

For the minimum t-norm the residuum and the negation look as follows

$$
x \rightarrow y=\left\{\begin{array}{ll}
1 & \text { if } x \leq y, \\
y & \text { if } x>y
\end{array} \quad \text { and } \quad \neg x= \begin{cases}1 & \text { if } x=0, \\
0 & \text { if } x>0 .\end{cases}\right.
$$


Let $T$ be a t-norm. The $T$-composition of two fuzzy relations $Q_{1}$ and $Q_{2}$ is denoted by $Q_{1} \circ_{T} Q_{2}$, and it is defined by $\left(Q_{1} \circ_{T} Q_{2}\right)(a, b)=\sup _{c \in X} T\left(Q_{1}(a, c), Q_{2}(c, b)\right)$ for all $a, b \in X$.

Definition 3.4. A fuzzy relation $Q$ is T-transitive if

$$
T(Q(a, b), Q(b, c)) \leq Q(a, c), \quad \text { for all } a, b, c \in X .
$$

The min-transitive closure of a fuzzy relation $Q$ is the smallest min-transitive fuzzy relation that contains $Q$. We denote it by $Q^{T}$, the capital $T$ standing for transitive compatible extension. As it is well known, $Q^{T}=\bigcap\left\{Q_{i} \supseteq Q \mid\right.$ $Q_{i}$ min-transitive $\}$.

The notion of completeness admits different generalizations too, and we consider its weakest form. We say that a fuzzy relation $Q$ is total if for all distinct $a, b \in X$ it holds that either $Q(a, b)>$ or $Q(b, a)>0$. Therefore a reflexive relation $R$ is total if and only if either $R(a, b)>0$ or $R(b, a)>0$ for all $a, b \in X$.

Definition 3.5. A fuzzy relation $R$ is a fuzzy preorder if it is reflexive and min-transitive.

Later on in this paper we discuss the connection between transitive-consistency and transitivity in the fuzzy context (cf., section 4 below).

Definition 3.6. A fuzzy relation $Q$ is $T$-acyclic if

$$
T\left(Q\left(a_{1}, a_{2}\right), Q\left(a_{2}, a_{3}\right), \ldots, Q\left(a_{n}, a_{1}\right)\right)=0, \quad \text { for all } a_{1}, \ldots, a_{n} \in X .
$$

Associated with fuzzy weak preference relations one can define strict preference components in several ways. Along this paper we fix the following notions: for each $a, b \in X$,

$$
I(a, b)=\min (R(a, b), R(b, a)), \quad P(a, b)= \begin{cases}R(a, b) & \text { if } R(b, a)=0, \\ 0 & \text { if } R(b, a)>0 .\end{cases}
$$

Given this (or any other) procedure for producing the strict component, Definitions 3.7 and 3.8 below are crucial in the rest of the paper:

Definition 3.7. A fuzzy weak preference relation $R$ with an associated strict preference relation $P$ is $T$-transitive-consistent if

$T\left(P\left(a_{1}, a_{2}\right), R\left(a_{2}, a_{3}\right), \ldots, R\left(a_{n-1}, a_{n}\right)\right) \leq \neg R\left(a_{n}, a_{1}\right) \quad$ for all $a_{1}, \ldots, a_{n} \in X$.

For the minimum t-norm, one has that $R$ is min-transitive-consistent if and only if for every $a_{1}, \ldots, a_{n} \in X$,

$$
\min \left(P\left(a_{1}, a_{2}\right), R\left(a_{2}, a_{3}\right), \ldots, R\left(a_{n-1}, a_{n}\right)\right) \leq 1-R\left(a_{n}, a_{1}\right) .
$$

This property is called Suzumura transitive-consistency in [17]. 
Definition 3.8. A fuzzy relation $\tilde{R}$ is an extension of the fuzzy relation $R$ on the set $X$ if and only if $R \subseteq \tilde{R}$.

A fuzzy weak preference relation $\tilde{R}$ with associated strict preference relation $\tilde{P}$ is a compatible extension of the fuzzy weak preference relation $R$ with associated strict preference relation $P$ on the set $X$ if and only if $R \subseteq \tilde{R}$ and $P \subseteq \tilde{P}$.

Given a left continuous $t$-norm $T$ and $\rightarrow$ its residuum, the fuzzy relation $\tilde{R}$ is a $T$-compatible extension of $R$ if $R \subseteq \tilde{R}$ and $\tilde{R}(b, a) \leq R(a, b) \rightarrow R(b, a)$ for all $a, b \in X$.

The relation $R$ is $T$-consistent if its $T$-transitive closure $R^{T}$ is a T-compatible extension of $R$.

The notion of $T$-compatible extension was introduced by Georgescu in [19]. Every min-compatible extension is a compatible extension but the opposite is not true as Example 4.6 in [19] shows. Here we give an alternative argument of a stronger result. Namely, the transitive closure of a min-transitive consistent fuzzy relation is a compatible extension of it, but it is not necessarily a mincompatible extension of the original relation as Example 4.1 below shows.

Unless otherwise stated, the results henceforth stated refer to the transitivity based on the minimum t-norm, i.e., to min-transitivity. Accordingly, for any fuzzy relation $Q$, min-transitivity means $Q \circ Q \subseteq Q$.

\section{Conditional fuzzy extensions}

Alcantud and Díaz [2, Remark 1] show that also in the fuzzy context, constraints cannot be imposed on the extensions of a fuzzy preorder with impunity. If we appeal to the general construction based on generators (cf., De Baets and Fodor [11]) in order to define the notion of a fuzzy extension through Definition 3.8, then [2] shows that Corollary 2.1 can be faithfully extended but Corollary 2.2 only admits a partial generalization. This raises the question whether other constructions can provide a full characterization accounting for the general case approached in Corollary 2.2.

Although generators have proven to be the most appropriate way to decompose fuzzy weak preference relations, other tighter constructions have been studied in the literature. In particular, Georgescu [17] proved several generalizations of Szpilrajn theorem via the construction in Eq. (6). Henceforth we assume that for any weak preference relation, its associated indifference and strict preference relations are defined by Eq. (6). The negative side of this approach is that these formulae do not determine the original weak preference relation $R$. However, we proceed to show that it performs very well when it comes to extending relations.

We proceed to prove the fuzzy counterparts of Corollaries 2.1 and 2.2. In particular we extend the characterization of Corollary 2.2 to the fuzzy set context. This shows that despite other drawbacks, the construction of Eq. (6) 
leads to better results in the context of extensions than the construction based on generators.

Firstly we consider the simpler case where we request that a number of elements are in strict relation with respective elements in the extended total preorder. This particular statement facilitates the subsequent proof of a more general result. To that purpose we need some previous results.

Although not every $T$-transitive-consistent relation is $T$-transitive, the following lemma proves a relationship between these properties:

Lemma 4.1. Let $T$ be a $t$-norm without zero divisors. Every $T$-transitive fuzzy weak preference relation $R$ on a universe $X$ is also T-transitive-consistent.

Proof. Assume that the $T$-transitive fuzzy relation $R$ is not $T$-transitive-consistent. Then there exist alternatives $a_{1}, \ldots, a_{n} \in X$ such that

$$
T\left(P\left(a_{1}, a_{2}\right), R\left(a_{2}, a_{3}\right), \ldots, R\left(a_{n-1}, a_{n}\right)>\neg R\left(a_{n}, a_{1}\right) .\right.
$$

Two implications derive.

Firstly, $R\left(a_{n}, a_{1}\right)>0$ because otherwise $\neg R\left(a_{n}, a_{1}\right)=1$, which contradicts the previous inequality. Secondly, $T\left(P\left(a_{1}, a_{2}\right), R\left(a_{2}, a_{3}\right), \ldots, R\left(a_{n-1}, a_{n}\right)>0\right.$. Since $T$ does not admit zero divisors, $\min \left(P\left(a_{1}, a_{2}\right), R\left(a_{2}, a_{3}\right), \ldots, R\left(a_{n-1}, a_{n}\right)>\right.$ 0 . By $T$-transitivity we deduce $R\left(a_{2}, a_{n}\right)>0$, which coupled with the fact that $R\left(a_{n}, a_{1}\right)>0$, implies $R\left(a_{2}, a_{1}\right)>0$. By the construction of $P$, the contradiction $P\left(a_{1}, a_{2}\right)=0$ follows.

Theorem 4.1 below crucially hinges on our maintained assumption that $P$ is deduced from Eq. (6).

Theorem 4.1. [19, Theorem 5.5] For a fuzzy relation $R$ on $X$ the following are equivalent:

1. $R$ has a total and min-transitive min-compatible extension $Q$.

2. $R$ has a min-transitive min-compatible extension $Q$.

3. $R$ is min-consistent.

Lemma 4.2. [19, Lemma 4.9] If $Q$ is a min-compatible extension of $R$ and $S$ is a min-compatible extension of $Q$, then $S$ is min-compatible extension of $R$.

As a direct corollary of Theorem 4.1, every min-transitive fuzzy relation $R$ has a total and min-transitive min-compatible extension. When the relation is reflexive we get:

Corollary 4.1. For every fuzzy preorder there is a fuzzy total preorder that is a min-compatible extension of it.

In particular, for every fuzzy preorder there is a fuzzy total preorder that is a compatible extension of it. 
Lemma 4.3. [17, Corollary 3.5] A fuzzy relation $R$ is min-transitive-consistent ${ }^{1}$ if and only if $R^{T}$ is a compatible extension of $R$ if and only if $P_{R} \subseteq P_{R^{T}}$ (where $P_{R^{T}}$ is the strict preference relation associated with the transitive closure $R^{T}$ of $R)$.

As a consequence, for any min-transitive-consistent fuzzy relation $R$ on $X$, any relation $Q$ that is a compatible extension of $R^{T}$ is also a compatible extension of $R$. The converse implication also holds under transitivity:

Lemma 4.4. Given a fuzzy min-transitive-consistent relation $R$, every mintransitive compatible extension of $R$ is a compatible extension of $R^{T}$.

Therefore, on the grounds of the construction of Eq. (6) we can state a fuzzy counterpart of Theorem 1 in [1].

Theorem 4.2. Let $R$ be a min-transitive fuzzy relation on $X$, and let $X_{I}=$ $\left\{a_{1}, \ldots, a_{n}, b_{1}, \ldots, b_{n}\right\}$ be a subset of possibly repeated elements of $X$. The following statements are equivalent:

a) There exists a total preorder $\tilde{R}$ that is a min-compatible extension of $R$ and such that $\tilde{P}\left(b_{i}, a_{i}\right)=1$ for all $i=1, \ldots, n$, where $\tilde{P}$ denotes the asymmetric part of $\tilde{R}$.

b) The relation $R^{A}$ associated with $X_{I}$ and $R$ is min-acyclic.

Proof. ${ }^{2}$ Let us assume that $a$ ) is true but $b$ ) does not hold. Then there exists a list of alternatives $a_{i_{1}}, \ldots, a_{i_{k}}$ such that

$$
R^{A}\left(a_{i_{1}}, a_{i_{2}}\right)>0, \quad R^{A}\left(a_{i_{2}}, a_{i_{3}}\right)>0, \quad \ldots \quad, R^{A}\left(a_{i_{k}}, a_{i_{1}}\right)>0,
$$

or equivalently,

$$
R\left(a_{i_{1}}, b_{i_{2}}\right)>0, \quad R\left(a_{i_{2}}, b_{i_{3}}\right)>0, \quad \ldots \quad, R\left(a_{i_{k}}, b_{i_{1}}\right)>0 .
$$

From the latter inequalities, because $\tilde{R}$ is an extension of $R$

$$
\tilde{R}\left(a_{i_{1}}, b_{i_{2}}\right)>0, \quad \tilde{R}\left(a_{i_{2}}, b_{i_{3}}\right)>0, \quad \ldots \quad, \tilde{R}\left(a_{i_{k}}, b_{i_{1}}\right)>0 .
$$

Also, $\tilde{P}\left(b_{i_{j}}, a_{i_{j}}\right)=1$ for all $j=1, \ldots, k$ by our assumption. Therefore because the inequalities

$$
\tilde{R}\left(a_{i_{1}}, b_{i_{2}}\right)>0, \quad \tilde{P}\left(b_{i_{2}}, a_{i_{2}}\right)>0, \quad \ldots \quad, \tilde{R}\left(a_{i_{k}}, b_{i_{1}}\right)>0
$$

have been established we obtain by min-transitivity that $\tilde{R}\left(a_{i_{1}}, b_{i_{1}}\right)>0$. This contradicts the fact that $\tilde{P}\left(b_{i_{1}}, a_{i_{1}}\right)=1$.

\footnotetext{
${ }^{1}$ Contrary to our notation, Georgescu calls transitive consistent to those fuzzy relations such that $R^{T}$ is a compatible extension of $R$.

${ }^{2}$ This proof partially replicates the proof of Theorem 2 in [2]. At the risk of being reiterative, we present all the steps for the sake of completeness. We insist that the formulae used here and in [2] to obtain the indifference and strict preference relations are different.
} 
Now let us assume that $b$ ) is true. In order to prove $a$ ) we proceed in several steps.

Define the relation $\bar{R}$ as follows: for each $a, b \in X$,

$$
\bar{R}(a, b)= \begin{cases}R(a, b) & \text { if }(a, b) \neq\left(b_{i}, a_{i}\right) \text { for all } i \\ 1 & \text { otherwise }\end{cases}
$$

Then we prove the following claim:

Claim. For each $a, b \in X$, if there exist $c_{1} \ldots, c_{k}$ alternatives in $X$ such that

$$
\min \left(\bar{R}\left(a, c_{1}\right), \ldots, \bar{R}\left(c_{k}, b\right)\right)>0
$$

then either

$$
R(a, b) \geq \min \left(\bar{R}\left(a, c_{1}\right), \ldots, \bar{R}\left(c_{k}, b\right)\right)
$$

or

$$
\exists\left\{j_{1}, \ldots, j_{l}\right\} \subseteq\{1, \ldots, n\} \mid \min \left(R\left(a, b_{j_{1}}\right), R\left(a_{j_{1}}, b_{j_{2}}\right), \ldots, R\left(a_{j_{l}}, b\right)\right)>0
$$

To prove the claim, let $c_{0}=a$ and $c_{k+1}=b$. We distinguish two cases.

If $\left(c_{i}, c_{i+1}\right) \neq\left(b_{j}, a_{j}\right)$ for any $j \in\{1, \ldots, n\}$, then $\bar{R}\left(c_{i}, c_{i+1}\right)=R\left(c_{i}, c_{i+1}\right)$ for all $i$ and since $R$ is min-transitive, it holds that $R(a, b) \geq \min \left(R\left(a, c_{1}\right), \ldots, R\left(c_{k}, b\right)\right)=$ $\min \left(\bar{R}\left(a, c_{1}\right), \ldots, \bar{R}\left(c_{k}, b\right)\right)$. This provides the statement in Eq. (8).

Otherwise there are $l$ indexes $\left\{i_{1}, \ldots, i_{l}\right\} \subseteq\{1, \ldots, k\}$ such that

$$
\begin{aligned}
& \left(c_{i_{1}}, c_{i_{1}+1}\right)=\left(b_{j_{1}}, a_{j_{1}}\right), \\
& \ldots, \\
& \left(c_{i_{l}}, c_{i_{l}+1}\right)=\left(b_{j_{l}}, a_{j_{l}}\right)
\end{aligned}
$$

for some $\left\{j_{i}, \ldots, j_{l}\right\} \in\{1, \ldots, n\}$,

$$
\begin{aligned}
R\left(c_{i_{1}+1}, c_{i_{2}}\right) & \geq \min \left(R\left(c_{i_{1}+1}, c_{i_{1}+2}\right), \ldots, R\left(c_{i_{2}-1}, c_{i_{2}}\right)\right) \\
& =\min \left(\bar{R}\left(c_{i_{1}+1}, c_{i_{1}+2}\right), \ldots, \bar{R}\left(c_{i_{2}-1}, c_{i_{2}}\right)\right)>0 .
\end{aligned}
$$

A similar reasoning establishes $R\left(c_{i_{2}+1}, c_{i_{3}}\right)>0, \ldots, R\left(c_{i_{l-1}+1}, c_{i_{l}}\right)>0$. Furthermore, if $a \neq c_{i_{1}}$ then $R\left(a, c_{i_{1}}\right) \geq \min \left(R\left(a, c_{1}\right), \ldots, R\left(c_{i_{1}-1}, c_{i_{1}}\right)\right)>0$ and if $c_{i_{l}} \neq b$ then $R\left(c_{i_{l}+1}, b\right)>0$. Thus we get

$$
\min \left(R\left(a, c_{i_{1}}\right), R\left(c_{i_{1}+1}, c_{i_{2}}\right), \ldots, R\left(c_{i_{l}+1}, b\right)\right)>0
$$

which can be expressed as follows:

$$
\min \left(R\left(a, b_{j_{1}}\right), R\left(a_{j_{1}}, b_{j_{2}}\right), \ldots, R\left(a_{j_{l}}, b\right)\right)>0 .
$$

This provides the statement in Eq. (9).

We claim that $\bar{R}^{T}$, the transitive closure of $\bar{R}$, is a min-compatible extension of $R$. It is clear that $R \subseteq \bar{R}^{T}$ because $R \subseteq \bar{R} \subseteq \bar{R}^{T}$. Fix $(a, b)$, we prove that $\bar{R}^{T}(b, a) \leq R(b, a) \rightarrow R(a, b)$. If $R(a, b) \leq R(b, a)$, then according to Eq. (5), $R(a, b) \rightarrow R(b, a)=1$ and the inequality holds trivially. Assume that 
$R(a, b)>R(b, a)$. If $\bar{R}^{T}(b, a)=R(b, a)$ then the inequality follows from Eq. (2) and we are done. Let us prove that $\bar{R}^{T}(b, a)>R(b, a)$ is absurd.

It must be the case that $\bar{R}(b, a)=R(b, a)$ because otherwise $(b, a)=\left(b_{i}, a_{i}\right)$ for some $i$ and $R\left(a_{i}, b_{i}\right)=R(a, b)>0$ contradicts the fact that $R^{A}$ is min-acyclic (for $R^{A}\left(a_{i}, a_{i}\right)>0$ ). Therefore one has $\bar{R}^{T}(b, a)>\bar{R}(b, a)$. By the definition of transitive closure, there exist $c_{1}, \ldots, c_{k}$ alternatives such that

$$
\min \left(\bar{R}\left(b, c_{1}\right), \bar{R}\left(c_{1}, c_{2}\right), \ldots, \bar{R}\left(c_{k}, a\right)\right)>\bar{R}(b, a) .
$$

By using the Claim above, the previous inequality leads to either

$$
R(b, a) \geq \min \left(\bar{R}\left(b, c_{1}\right), \ldots, \bar{R}\left(c_{k}, a\right)\right)
$$

or

$$
\exists\left\{i_{1}, \ldots, i_{l}\right\} \subseteq\{1, \ldots, n\} \mid \min \left(R\left(b, b_{i_{1}}\right), R\left(a_{i_{1}}, b_{i_{2}}\right), \ldots, R\left(a_{i_{l}}, a\right)\right)>0 .
$$

The first possibility produces the contradiction

$$
R(b, a) \geq \min \left(\bar{R}\left(b, c_{1}\right), \bar{R}\left(c_{1}, c_{2}\right), \ldots, \bar{R}\left(c_{k}, a\right)\right)>\bar{R}(b, a) .
$$

Now assume the second possibility. Then

$$
\left.\min \left(R\left(a_{i_{1}}, b_{i_{2}}\right)\right), R\left(a_{i_{2}}, b_{i_{3}}\right), \ldots, R\left(a_{i_{l}}, b_{i_{1}}\right)\right)>0,
$$

or equivalently,

$$
\left.\min \left(R^{A}\left(a_{i_{1}}, a_{i_{2}}\right)\right), R^{A}\left(a_{i_{2}}, a_{i_{3}}\right), \ldots, R^{A}\left(a_{i_{l}}, a_{i_{1}}\right)\right)>0,
$$

which contradicts the fact that $R^{A}$ is min-acyclic.

Let us prove that $\bar{P}^{T}\left(b_{i}, a_{i}\right)=1$ for all $i \in\{1, \ldots, n\}$. Fix an arbitrary $i \in\{1, \ldots, n\}$. Since $\bar{R}^{T}\left(b_{i}, a_{i}\right) \geq \bar{R}\left(b_{i}, a_{i}\right)=1$, it only remains to prove that $\bar{R}^{T}\left(a_{i}, b_{i}\right)=0$. It is clear that $R\left(a_{i}, b_{i}\right)=0$, otherwise $R^{A}\left(a_{i}, a_{i}\right)>0$ against the fact that $R^{A}$ is min-acyclic. Furthermore $\bar{R}\left(a_{i}, b_{i}\right)=0$, otherwise $R\left(a_{i}, b_{i}\right) \neq \bar{R}\left(a_{i}, b_{i}\right)$ thus necessarily $\left(a_{i}, b_{i}\right)=\left(b_{j}, a_{j}\right)$ for some $j$, and because $R^{T}$ is reflexive

$$
\begin{aligned}
& 1=R\left(a_{i}, a_{i}\right)=R\left(a_{i}, b_{j}\right)=R^{A}\left(a_{i}, a_{j}\right), \\
& 1=R\left(a_{j}, a_{j}\right)=R\left(a_{j}, b_{i}\right)=R^{A}\left(a_{j}, a_{i}\right),
\end{aligned}
$$

against the fact that $R^{A}$ is min-acyclic.

Now, if $\bar{R}^{T}\left(a_{i}, b_{i}\right)>0$ then

$$
\min \left(\bar{R}\left(a_{i}, c_{1}\right), \ldots, \bar{R}\left(c_{k}, b_{i}\right)\right)>0 .
$$

So the Claim above applies again. Eq. (8) contradicts $R\left(a_{i}, b_{i}\right)=0$. If Eq. (9) applies, then there exists a set $\left\{j_{1}, \ldots, j_{l}\right\} \in\{1, \ldots, n\}$ such that

$$
\min \left(R\left(a_{i}, b_{j_{1}}\right), R\left(a_{j_{1}}, b_{j_{2}}\right), \ldots, R\left(a_{j_{l}}, b_{i}\right)\right)>0 .
$$


Equivalently, $\min \left(R^{A}\left(a_{i}, a_{j_{1}}\right), R^{A}\left(a_{j_{1}}, a_{j_{2}}\right), \ldots, R^{A}\left(a_{j_{l}}, a_{i}\right)\right)>0$, a cycle in $R^{A}$, which is absurd.

From $\bar{R}^{T}\left(a_{i}, b_{i}\right)=0$ and $\bar{R}^{T}\left(b_{i}, a_{i}\right)=1$, one has $\bar{P}^{T}\left(b_{i}, a_{i}\right)=\bar{R}^{T}\left(b_{i}, a_{i}\right)=1$.

Since $\bar{R}^{T}$ is transitive, Theorem 4.1 assures that there exists a total preorder $\tilde{R}$ that is a min-compatible extension of $\bar{R}^{T}$. Then $\tilde{P}\left(b_{i}, a_{i}\right)=1$ for all $i \in$ $\{1, \ldots, n\}$. Furthermore, because $\tilde{R}$ is a min-compatible extension of $\bar{R}^{T}$ which is a min-compatible extension of $R$, then it follows from Lemma 4.2 that $\tilde{R}$ is a min-compatible extension of $R$. This ends the proof.

Corollary 4.2. Let $R$ be a min-transitive-consistent fuzzy relation on $X$, and let $X_{I}=\left\{a_{1}, \ldots, a_{n}, b_{1}, \ldots, b_{n}\right\}$ be a subset of possibly repeated elements of $X$. The following statements are equivalent:

a) There exists a total preorder $\tilde{R}$ that is a compatible extension of $R$ and such that $\tilde{P}\left(b_{i}, a_{i}\right)=1$ for all $i=1, \ldots, n$, where $\tilde{P}$ denotes the asymmetric part of $\tilde{R}$.

b) The relation $R^{A}$ associated with $X_{I}$ and $R^{T}$, where $R^{T}$ is the min-transitive closure of $R$, is min-acyclic.

Proof. Assume $a$ ) is true. To prove that $b$ ) holds we can copy the steps followed in the proof of Theorem 4.2.

The converse implication follows from Theorem 4.2 and Lemma 4.1.

This corollary proves that min-transitive-consistency is a sufficient condition for the existence of a compatible extension satisfying certain conditions. However, it is not a sufficient condition if we want to obtain a min-compatible extension as the following example proves:

Example 4.1. Consider the fuzzy relation $R$ defined on $X=\{a, b, c\}$ as follows and its associated strict preference relation $P$.

\begin{tabular}{c|ccc}
$R$ & $a$ & $b$ & $c$ \\
\hline$a$ & 1 & 0.7 & 0.7 \\
$b$ & 0.7 & 1 & 0.7 \\
$c$ & 0.5 & 0.7 & 1
\end{tabular}

\begin{tabular}{c|ccc}
$P$ & $a$ & $b$ & $c$ \\
\hline$a$ & 0 & 0 & 0 \\
$b$ & 0 & 0 & 0 \\
$c$ & 0 & 0 & 0
\end{tabular}

Since $P\left(d_{1}, d_{2}\right)=0$ for all $d_{1}, d_{2} \in X$, it is easy to check that $R$ is min-transitiveconsistent. But there exists no min-transitive relation $\tilde{R}$ that is a min-compatible extension of $R$. Such an $\tilde{R}$ must satisfy

$$
\tilde{R}(c, a) \geq \min (\tilde{R}(c, b), \tilde{R}(b, a)) \geq \min (R(c, b), R(b, a)) .
$$

Thus, $\tilde{R}(c, a) \geq 0.7$. However, this means that $\tilde{R}$ cannot be a min-compatible extension of $R$ :

$$
\tilde{R}(c, a) \geq 0.7>0.5=0.7 \rightarrow 0.5=R(a, c) \rightarrow R(c, a) .
$$


Theorem 4.2 extends Theorem 1 in [1]. In spirit this achievement can be compared to Theorem 2 in [2]. Corollary 4.2 extends Corollary 2.1. They concern a more general class of relations, namely min-transitive-consistent relations, whereas in Theorem 4.2 and [2, Theorem 2] the original relation must be transitive.

Now we proceed to consider the more general case where some elements are asked to be in strict relation with respective elements in the extended total preorder, while some other are asked to be indifferent (i.e., with full relationship) to respective companions. This ultimately yields a fuzzy counterpart of Corollary 2.2 and [1, Theorem 2]. To prove it we establish Lemma 4.5 below and then introduce some definitions.

Lemma 4.5. Let $R$ be a fuzzy reflexive relation defined on $X$. Then ${ }^{3}$

$$
R \circ R \subseteq R \quad \Rightarrow \quad\left\{\begin{array}{l}
P \circ P \subseteq P \\
P \circ I \subseteq P \\
I \circ P \subseteq P .
\end{array}\right.
$$

Proof. In order to prove min-transitivity of $P$, assume $P(a, b)=x>0$ and $P(b, c)=y>0$. Then $R(a, b)=x, R(b, c)=y$ and $R(b, a)=R(c, b)=0$. This implies that $R(a, c) \geq \min (x, y)$ by min-transitivity of $R$. If $R(c, a)>0$ then we have $R(c, b) \geq \min (R(c, a), R(a, b))>0$, a contradiction, thus $P(a, c)=$ $R(a, c) \geq \min (x, y)$.

In order to prove $\min (P(a, b), I(b, c)) \leq P(a, c)$ for each $a, b, c \in X$, we assume $P(a, b)=x>0$ and $I(b, c)=y>0$. Then $R(a, b)=x, \min (R(b, c), R(c, b))=$ $y$, and $R(b, a)=0$. This implies $R(a, c) \geq \min (x, y)$. Now, if $R(c, a)>0$ then $R(b, a)>0$, a contradiction that completes the argument.

The case $\min (I(a, b), P(b, c)) \leq P(a, c)$ for each $a, b, c \in X$ is similar.

Let $X_{I}^{n}=\left\{a_{n+1}, \ldots, a_{p}, b_{n+1}, \ldots, b_{p}\right\}$. If $n=p$, then $X_{I}^{n}=\varnothing$.

Definition 4.1. The relation $R^{I}$ associated with $R$ and $X_{I}$ is defined as follows:

$$
R^{I}(a, b)=R(a, \delta(b)), \text { for all } a, b \in X_{I} .
$$

where $\delta(b)$ is the function introduced in Definition 2.6.

Definition 4.2. The relation $R^{G}$ associated with $R, n \leq p$ and $X_{I}$ is defined by

$R^{G}\left(a_{i}, a_{j}\right)=\max \left(R\left(a_{i}, b_{j}\right), \sup _{\left\{c_{1}, \ldots c_{k}\right\} \subseteq X_{I}^{n}}\left(\min \left(R\left(a_{i}, \delta\left(c_{1}\right)\right), R\left(c_{1}, \delta\left(c_{2}\right)\right), \ldots, R\left(c_{k}, b_{j}\right)\right)\right)\right)$.

Definition 4.3. The relation $R^{G}$ is $\delta$-consistent with $X_{I}$ and $n \leq p$ if $\min \left(R^{G}\left(a_{i_{1}}, a_{i_{2}}\right), \ldots, R^{G}\left(a_{i_{k}}, a_{i_{1}}\right)\right)>0$ implies $i_{t}>n$ for all $t \in\{1, \ldots, k\}$.

\footnotetext{
${ }^{3}$ This implication was studied by Dasgupta and Deb [10] and Díaz et al. [13, 14] for constructions unrelated to Eq. (6).
} 
In [2] the following Proposition 4.1 is proven in a context where the strict preference and indifference relations associated with a weak preference relation are obtained using a generator. A careful look at the proof reveals that the result holds true independently of the construction considered and in particular, it applies under our maintained assumption that Eq. (6) is used to derive strict preference and indifference relations from weak preference relations:

Proposition 4.1 (Alcantud and Díaz [2], Prop. 2). Let $R$ be a fuzzy preorder. If there exists a fuzzy total preorder $\tilde{R}$ that is a compatible extension of $R$, such that $\tilde{P}\left(b_{i}, a_{i}\right)=1$ for each $i=1, \ldots, n$ and $\tilde{I}\left(b_{i}, a_{i}\right)=1$ for $i=n+1, \ldots, p$, then the relation $R^{G}$ is $\delta$-consistent with $X_{I}$ and $n$.

We will provide two extensions of Corollary 2.2 thus [1, Theorem 2]. In order to prove the first one, Theorem 4.3, we introduce the notion of (fuzzy) $\delta$-cyclicity. The proof of Theorem 4.4 involves a stronger concept that we will introduce later.

Definition 4.4. We say that $R^{I}$ is $\delta$-cyclic along $X_{I}^{n}$ if $R^{I}\left(c_{1}, c_{2}\right)>0$ and $R^{I}\left(c_{2}, c_{3}\right)>0$ and $\ldots R^{I}\left(c_{k}, c_{1}\right)>0$ implies that

$$
R^{I}\left(\delta\left(c_{1}\right), \delta\left(c_{k}\right)\right)>0, \quad \ldots \quad, R^{I}\left(\delta\left(c_{2}\right), \delta\left(c_{1}\right)\right)>0, \text { for all } c_{1}, \ldots, c_{k} \in X_{I}^{n} .
$$

Theorem 4.3. Assume that for any fuzzy weak preference relation the associated strict preference and indifference relations are defined as in $E q$. (6). Let $R$ be a min-transitive-consistent fuzzy relation on $X$. Let $X_{I}=\left\{a_{1}, \ldots, a_{p}, b_{1}, \ldots, b_{p}\right\}$ be an ordered list of possibly repeated elements of $X$ and let $n \leq p$. The following statements are equivalent:

a) There exists a fuzzy total preorder $\tilde{R}$ that is a compatible extension of $R$ such that $\tilde{P}\left(b_{i}, a_{i}\right)=1$ for each $i=1, \ldots, n$ and $\tilde{I}\left(b_{i}, a_{i}\right)=1$ for $i=n+1, \ldots, p$.

b) The relation $R^{G}$ associated with $R^{T}$ is $\delta$-consistent with $X_{I}$ and $n$, and $R^{I}$ (associated with $R^{T}$ ) is $\delta$-cyclic along $X_{I}^{n}$.

Proof. Let us assume that $a$ ) is true. According to Lemma 4.4, the relation $\tilde{R}$ is a compatible extension of $R^{T}$. It follows from Proposition 4.1 that the relation $R^{G}$ is $\delta$-consistent with $X_{I}$ and $n$.

In order to prove that $R^{I}$ is $\delta$-cyclic along $X_{I}^{n}$, let us assume that $c_{1}, \ldots c_{k} \in$ $X_{I}^{n}$ are such that

$$
\min \left(R^{I}\left(c_{1}, c_{2}\right), \ldots, R^{I}\left(c_{k}, c_{1}\right)\right)>0 \quad \text { but } R^{I}\left(\delta\left(c_{j+1}\right), \delta\left(c_{j}\right)\right)=0
$$

for some $j \in\{1, \ldots, k\}$ with the convention $c_{k+1}=c_{1}$. From this we obtain the equality $R\left(\delta\left(c_{j+1}\right), c_{j}\right)=0$. Since $R^{I}\left(c_{j}, c_{j+1}\right)>0$ and this means $R\left(c_{j}, \delta\left(c_{j+1}\right)\right)>0$, we deduce $P\left(c_{j}, \delta\left(c_{j+1}\right)\right)>0$ which in turn implies $\tilde{P}\left(c_{j}, \delta\left(c_{j+1}\right)\right)>$ 0 . In addition,

$$
\min \left(\tilde{I}\left(\delta\left(c_{j+1}\right), c_{j+1}\right), \tilde{R}\left(c_{j+1}, \delta\left(c_{j+2}\right)\right), \tilde{I}\left(\delta\left(c_{j+2}\right), c_{j+2}\right), \ldots, \tilde{I}\left(\delta\left(c_{j}\right), c_{j}\right)\right)>0 .
$$


Since $\tilde{R}$ is min-transitive, this leads to $\tilde{R}\left(\delta\left(c_{j+1}\right), c_{j}\right)>0$. We obtain the contradiction: $\tilde{P}\left(c_{j}, \delta\left(c_{j+1}\right)\right)>0$ and $\tilde{R}\left(\delta\left(c_{j+1}\right), c_{j}\right)>0$.

Now let us assume that $b$ ) is true. We use the properties of the auxiliary relation $\bar{R}$ defined as follows:

$$
\begin{aligned}
\bar{R}(a, b) & =\max \left(R^{T}(a, b),\right. \\
& \left.\sup _{\left\{d_{1}, \ldots d_{k}\right\} \subseteq X_{I}^{n}}\left(\min \left(R^{T}\left(a, \delta\left(d_{1}\right)\right), R^{I}\left(d_{1}, d_{2}\right), \ldots, R^{I}\left(d_{k-1}, d_{k}\right), R^{T}\left(d_{k}, b\right)\right)\right)\right) .
\end{aligned}
$$

Then $\bar{R}\left(a_{i}, b_{j}\right)=R^{G}\left(a_{i}, a_{j}\right)$ for all $i, j$.

It is easy to check that $\bar{R}$ is reflexive and min-transitive. Let $\bar{I}$ be the symmetric part of $\bar{R}$, then the fact that $\bar{I}\left(a_{i}, b_{i}\right)=1$ for all $i=n+1, \ldots, p$ follows from

$$
\begin{aligned}
& \bar{R}\left(a_{i}, b_{i}\right) \geq \min \left(R^{T}\left(a_{i}, \delta\left(b_{i}\right)\right), R^{T}\left(b_{i}, b_{i}\right)\right)=1 \quad \text { for each } i \\
& \bar{R}\left(b_{i}, a_{i}\right) \geq \min \left(R^{T}\left(b_{i}, \delta\left(a_{i}\right)\right), R^{T}\left(a_{i}, a_{i}\right)\right)=1 \quad \text { for each } i .
\end{aligned}
$$

Furthermore, $\bar{R}$ is a compatible extension of $R^{T}$. It is clear that $R^{T} \subseteq \bar{R}$. To prove $P^{T} \subseteq \bar{P}$ we fix arbitrary $a, b \in X$, then we need to assure $\bar{P}(a, b) \geq$ $P^{T}(a, b)$. Two cases arise. If $\bar{R}(b, a)=0$ then $\bar{P}(a, b)=\bar{R}(a, b) \geq R^{T}(a, b) \geq$ $P^{T}(a, b)$ and we are done. If $\bar{R}(b, a)>0$ we get a contradiction under the assumption $P^{T}(a, b)>\bar{P}(a, b)$ as follows. Observe that now $R^{T}(a, b)>0$, $R^{T}(b, a)=0$ and $\bar{R}(a, b)>0$ hold true. From $\bar{R}(b, a)>0$ we deduce the existence of $d_{1}, \ldots, d_{k} \in X_{I}^{n}$ such that

$$
\min \left(R^{I}\left(d_{1}, d_{2}\right), \ldots, R^{I}\left(d_{k-1}, d_{k}\right)\right)>0
$$

and also $R^{T}\left(b, \delta\left(d_{1}\right)\right)>0$ and $R^{T}\left(d_{k}, a\right)>0$. Now

$R^{T}\left(d_{k}, a\right)>0, R^{T}(a, b)>0, R^{T}\left(b, \delta\left(d_{1}\right)\right)>0 \quad$ imply $\quad R^{T}\left(d_{k}, \delta\left(d_{1}\right)\right)>0$.

So that $R^{I}\left(d_{1}, d_{2}\right)>0, R^{I}\left(d_{2}, d_{3}\right)>0, \ldots, R^{I}\left(d_{k}, d_{1}\right)>0$. Therefore an appeal to $b$ ) produces

$$
R^{I}\left(\delta\left(d_{1}\right), \delta\left(d_{k}\right)\right)>0, \ldots, R^{I}\left(\delta\left(d_{2}\right), \delta\left(d_{1}\right)\right)>0 .
$$

Then we get a contradiction that completes the argument because

$$
R^{T}\left(b, \delta\left(d_{1}\right)\right)>0, R^{T}\left(\delta\left(d_{1}\right), d_{k}\right)>0, R^{T}\left(d_{k}, a\right)>0 \text { imply } R^{T}(b, a)>0 .
$$

Hence $\bar{R}$ is a compatible extension of $R^{T}$. By Lemma 4.4, $\bar{R}$ is a compatible extension of $R$.

Now we are ready to conclude. If $n=0$ then any fuzzy total preorder $\tilde{R}$ that is a compatible extension of $\bar{R}$ is a compatible extension of $R$ too. If $n>0$ then we can apply Theorem 4.2: the relation $\bar{R}^{A}$ is min-acyclic on ${ }^{n} X_{I}=\left\{a_{1}, \ldots, a_{n}, b_{1}, \ldots, b_{n}\right\}$ since $R^{G}$ is $\delta$-consistent. Therefore there exists a total preorder $\tilde{R}$ that extends $\bar{R}$ such that $\tilde{P}\left(b_{i}, a_{i}\right)=1$ for $i \leq n$. In addition to this, $\tilde{I}\left(b_{i}, a_{i}\right)=1$ for all $i \geq n+1$ because $\tilde{R}$ extends $\bar{R}$ and $\bar{I}\left(a_{i}, b_{i}\right)=1$ for all $i \geq n+1$. This ends the proof. 
A comparison with the characterization in Alcantud and Díaz [2, Theorem 5] is in order. That result attempted to extend Corollary 2.2 too. To be precise, there it is shown that when we use generators in order to define the notion of a compatible extension, only a partial generalization can be proven: sufficient conditions for the existence of the desired extension, conditional on a given list of pairwise comparisons, are put forward. One of these conditions, namely, $\delta$ min-cyclicity, is not necessary with the construction by Eq. (6). Theorem 4.3 shows that by weakening this condition to $\delta$-cyclicity, a full characterization can be proven under the construction by Eq. (6) which therefore constitutes a fuzzy generalization of Corollary 2.2 .

However, if we want to assure the existence of a min-compatible extension, the departing relation $R$ has to be min-transitive and its associated $R^{I}, \delta$-mincyclic.

Definition 4.5. We say that $R^{I}$ is $\delta$-min-cyclic along $X_{I}^{n}$ if

$$
\begin{aligned}
& \min \left(R^{I}\left(a_{1}, a_{2}\right), R^{I}\left(a_{2}, a_{3}\right), \ldots, R^{I}\left(a_{k}, a_{1}\right)\right) \\
& \quad=\min \left(R^{I}\left(\delta\left(a_{1}\right), \delta\left(a_{k}\right)\right), \ldots R^{I}\left(\delta\left(a_{2}\right), \delta\left(a_{1}\right)\right)\right), \text { for all } a_{1}, \ldots, a_{k} \in X_{I}^{n} .
\end{aligned}
$$

Theorem 4.4. Assume that for any fuzzy weak preference relation the associated strict preference and indifference relations are defined as in Eq. (6). Let $R$ be a min-transitive fuzzy relation on $X$. Let $X_{I}=\left\{a_{1}, \ldots, a_{p}, b_{1}, \ldots, b_{p}\right\}$ be an ordered list of possibly repeated elements of $X$ and let $n \leq p$. The following statements are equivalent:

a) There exists a fuzzy total preorder $\tilde{R}$ that is a min-compatible extension of $R$ such that $\tilde{P}\left(b_{i}, a_{i}\right)=1$ for each $i=1, \ldots, n$ and $\tilde{I}\left(b_{i}, a_{i}\right)=1$ for $i=n+1, \ldots, p$.

b) The relation $R^{G}$ associated with $R$ is $\delta$-consistent with $X_{I}$ and $n$, and $R^{I}$ is $\delta$-min-cyclic along $X_{I}^{n}$.

Proof. The proof is very similar to the proof of Theorem 4.3.

Let us prove that $a$ ) implies $b$ ). Since $R$ is min-transitive, $R=R^{T}$ and since $\tilde{R}$ is a min-compatible extension of $R$, it is a compatible extension of $R$. Then it follows from Proposition 4.1 that $R^{G}$ is $\delta$-consistent with $X_{I}$ and $n$. Let us prove now that $R^{I}$ is $\delta$-min-cyclic. It holds that $\tilde{R}\left(a_{i}, b_{i}\right)=\tilde{R}\left(b_{i}, a_{i}\right)=$ 1 for $i=n+1, \ldots, p$. Since $\tilde{R}$ is a min-compatible extension of $R$, then $\tilde{R}(b, a) \leq R(a, b) \rightarrow R(b, a)$ for all $a, b$. This implies that $R\left(b_{i}, a_{i}\right) \leq R\left(a_{i}, b_{i}\right)$ and $R\left(a_{i}, b_{i}\right) \leq R\left(b_{i}, a_{i}\right)$, or equivalently, $R\left(b_{i}, a_{i}\right)=R\left(a_{i}, b_{i}\right)$ for $i=n+1, \ldots, p$. Now assume that $R^{I}$ is not $\delta$-min-cyclic. Then there exist $a_{1}, \ldots, a_{k} \in X_{I}^{n}$ such that

$$
\begin{aligned}
& \min \left(R^{I}\left(a_{1}, a_{2}\right), R^{I}\left(a_{2}, a_{3}\right), \ldots, R^{I}\left(a_{k}, a_{1}\right)\right) \\
& \quad<\min \left(R^{I}\left(\delta\left(a_{1}\right), \delta\left(a_{k}\right)\right), \ldots R^{I}\left(\delta\left(a_{2}\right), \delta\left(a_{1}\right)\right)\right) .
\end{aligned}
$$

Equivalently,

$$
\begin{aligned}
\min & \left(R\left(a_{1}, b_{2}\right), R\left(a_{2}, b_{3}\right), \ldots, R\left(a_{k}, b_{1}\right)\right) \\
\quad & <\min \left(R\left(b_{1}, a_{k}\right), R\left(b_{k}, a_{k-1}\right), \ldots, R\left(b_{3}, a_{2}\right), R\left(b_{2}, a_{1}\right)\right) .
\end{aligned}
$$


Then we obtain a contradiction since there exists some $i \in\{1, \ldots, k\}$ such that

$$
R\left(a_{i}, b_{i+1}\right)=\min \left(R\left(a_{1}, b_{2}\right), R\left(a_{2}, b_{3}\right), \ldots, R\left(a_{k}, b_{1}\right)\right)<R\left(b_{i+1}, a_{i}\right) .
$$

Now let us assume that $b$ ) is true. We use the properties of the auxiliary relation $\bar{R}$ defined as in the proof of Theorem 4.3. Let us recall that this relation is reflexive and min-transitive and $\bar{I}\left(a_{i}, b_{i}\right)=1$ for all $i=n+1, \ldots, p$.

Furthermore, $\bar{R}$ is a min-compatible extension of $R$. It is clear that $R^{T} \subseteq \bar{R}$. Let us prove that $\bar{R}(b, a) \leq R(a, b) \rightarrow R(b, a)$ for all $a, b \in X$. For any fixed $a, b \in X$, if $R(a, b) \leq R(b, a), R(a, b) \rightarrow R(b, a)=1$ and the inequality holds trivially.

Then assume $R(a, b)>R(b, a)$. If $\bar{R}(b, a)=R(b, a)$, the inequality also holds. Let us assume that $\bar{R}(b, a)>R(b, a)$, we will obtain a contradiction.

According to the definition of $\bar{R}$, there exist $d_{1}, \ldots, d_{k} \in X_{I}^{n}$ such that

$\bar{R}(b, a)=\min \left(R\left(b, \delta\left(d_{1}\right)\right), R^{I}\left(d_{1}, d_{2}\right), \ldots, R^{I}\left(d_{k-1}, d_{k}\right), R\left(d_{k}, a\right)\right)>R(b, a) \geq 0$.

Now $R^{I}\left(d_{k}, d_{1}\right)=R\left(d_{k}, \delta\left(d_{1}\right)\right) \geq \min \left(R\left(d_{k}, a\right), R(a, b), R\left(b, \delta\left(d_{1}\right)\right)\right)$. Then

$$
\begin{aligned}
& \min \left(R^{I}\left(d_{1}, d_{2}\right), \ldots, R^{I}\left(d_{k-1}, d_{k}\right), R^{I}\left(d_{k}, d_{1}\right)\right) \geq \\
& \quad \min \left(R^{I}\left(d_{1}, d_{2}\right), \ldots, R^{I}\left(d_{k-1}, d_{k}\right), R\left(d_{k}, a\right), R(a, b), R\left(b, \delta\left(d_{1}\right)\right)\right) .
\end{aligned}
$$

Since $R^{I}$ is $\delta$-min-cyclic, also

$$
\begin{aligned}
& \min \left(R^{I}\left(\delta\left(d_{1}\right), \delta\left(d_{k}\right)\right), \ldots, R^{I}\left(\delta\left(d_{2}\right), \delta\left(d_{1}\right)\right)\right) \geq \\
& \quad \min \left(R^{I}\left(d_{1}, d_{2}\right), \ldots, R^{I}\left(d_{k-1}, d_{k}\right), R\left(d_{k}, a\right), R(a, b), R\left(b, \delta\left(d_{1}\right)\right)\right) .
\end{aligned}
$$

In particular,

$$
R\left(\delta\left(d_{1}\right), d_{k}\right) \geq \min \left(R^{I}\left(d_{1}, d_{2}\right), \ldots, R^{I}\left(d_{k-1}, d_{k}\right), R\left(d_{k}, a\right), R(a, b), R\left(b, \delta\left(d_{1}\right)\right)\right) .
$$

Applying min-transitivity,

$$
\begin{aligned}
R(b, a) & \geq \min \left(R\left(b, \delta\left(d_{1}\right)\right), R\left(\delta\left(d_{1}\right), d_{k}\right), R\left(d_{k}, a\right)\right) \\
& \geq \min \left(R\left(b, \delta\left(d_{1}\right)\right), R^{I}\left(d_{1}, d_{2}\right), \ldots, R^{I}\left(d_{k-1}, d_{k}\right), R\left(d_{k}, a\right), R(a, b)\right) .
\end{aligned}
$$

Finally, since $R(b, a)<R(a, b)$, we get the contradiction

$$
R(b, a) \geq \min \left(R\left(b, \delta\left(d_{1}\right)\right), R^{I}\left(d_{1}, d_{2}\right), \ldots, R^{I}\left(d_{k-1}, d_{k}\right), R\left(d_{k}, a\right)\right)=\bar{R}(b, a) .
$$

The final part of this proof is similar to the proof of Theorem 4.3.

If $n=0$, since $\bar{R}$ is min-transitive, it follows from Theorem 4.1 that there exists a fuzzy total preorder $\tilde{R}$ that is a min-compatible extension of $\bar{R}$ and by Lemma 4.2 it is also a min-compatible extension of $R$. If $n>0$ then we can apply Theorem 4.2: the relation $\bar{R}^{A}$ is min-acyclic on ${ }^{n} X_{I}=\left\{a_{1}, \ldots, a_{n}, b_{1}, \ldots, b_{n}\right\}$ since $R^{G}$ is $\delta$-consistent. Therefore there exists a total preorder $\tilde{R}$ that is a min-compatible extension of $\bar{R}$ such that $\tilde{P}\left(b_{i}, a_{i}\right)=1$ for $i \leq n$. In addition to this, $\tilde{I}\left(b_{i}, a_{i}\right)=1$ for all $i \geq n+1$ because $\tilde{R}$ extends $\bar{R}$ and $\bar{I}\left(a_{i}, b_{i}\right)=1$ for all $i \geq n+1$. This ends the proof. 
We have characterized the existence of total preorders that satisfy some additional conditions and that are compatible extensions or min-compatible extensions of the original relation $R$. Theorem 4.4 shows that in order to obtain a min-compatible extension, the original relation has to be transitive and the associated relation $R^{I}$ has to be $\delta$-min-cyclic. Theorem 4.3 shows that a compatible extension can be found for a wider family of relations: on the one hand the departing relation has to be min-transitive consistent, not necessarily mintransitive, and on the other hand, it suffices to check that the associated $R^{I}$ is $\delta$-cyclic and not necessarily $\delta$-min-cyclic.

\section{Representing fuzzy relations as intersections of fuzzy total relations}

This Section has two objectives. It aims at proving some results on the representation of fuzzy relations, a task that we accomplish by first exploring the problem of extending fuzzy relations with fixed degrees of relation between pairs of elements. But it also shows that all these types of results can be expressed in terms of numerical indicators which capture the degree to which a fuzzy relation verifies a given property (cf., Belohlavek [4], Georgescu [18, 20, 21] and Gottwald [22]). ${ }^{4}$ In particular, we are concerned with the following indicators:

Definition 5.1. Given a fuzzy relation $R$ defined on $X$,

$$
\begin{aligned}
& \operatorname{Ref}(R)=\bigwedge_{a \in X} R(a, a) ; \\
& \operatorname{Trans}(R)=\bigwedge_{a, b, c \in X}[(R(a, c) \wedge R(c, b)) \rightarrow R(a, b)] ; \\
& \operatorname{Preord}(R)=\operatorname{Ref}(R) \wedge \operatorname{Trans}(R) .
\end{aligned}
$$

$\operatorname{Re} f(R)$ is called the degree of reflexivity of $R, \operatorname{Trans}(R)$ is called the degree of min-transitivity of $R$, and $\operatorname{Preord}(R)$ is interpreted as the degree to which $R$ is a fuzzy preorder. From the fuzzy logic point of view, $\operatorname{Re} f(R)$ (resp., $\operatorname{Trans}(R)$, $\operatorname{Preord}(R)$ ) measures the degree of truth of the statement "the relation $R$ is reflexive" (resp., min-transitive, fuzzy preorder).

Georgescu [18, Chapter 4.4] is concerned with the analysis of properties and indicators of fuzzy relations. As to the use of indicators in contexts related to our targets, we mention that Georgescu [20, Theorem 8] constitutes a generalization of the Szpilrajn theorem stated in terms of indicators. It generalizes a theorem in Bodenhofer and Klawonn [5] which already generalizes the Szpilrajn theorem.

\subsection{Fuzzy extensions with fixed degrees of relation}

Höhle and Blanchard [25, Theorem II.7] prove that also in the fuzzy setting, 'partial orders' (reflexive, antisymmetric, transitive) can be extended to linear

\footnotetext{
${ }^{4}$ We are very grateful to an anonymous referee for pointing out this stimulating possibility for research.
} 
orders with the restriction that a fixed pair of elements is in the same degree of relation before and after the extending process. From this result they state that a particularization of Szpilrajn's theorem where non-trivial indifferences are not allowed is a direct consequence (cf., [25, Corollary II.7']).

In this subsection we prove that a similar result holds true when the statement for the more general class of fuzzy preorders is invoked. In fact we derive this consequence from Proposition 5.1 below, which affects all fuzzy relations, by the appeal to indicators in the following terms:

Proposition 5.1. Given a fuzzy relation $R$ defined on a set $X$, let us fix $a_{0}, b_{0} \in X$ such that $R\left(a_{0}, b_{0}\right)=\alpha$ and $\beta>0$. Then there exists a fuzzy total relation $\tilde{R}$ that is a compatible extension of $R$ satisfying that $\operatorname{Re} f(R) \leq$ $\operatorname{Re} f(\tilde{R})$ and $\operatorname{Trans}(R) \leq \operatorname{Trans}(\tilde{R})$ and such that $\tilde{R}\left(a_{0}, b_{0}\right)=R\left(a_{0}, b_{0}\right)$ and $\tilde{R}(a, b)=R(a, b)$ for all $a, b$ with $R(a, b) \geq \beta$.

Furthermore, we can impose $\tilde{R}(a, b) \leq \beta$ when $R(a, b)<\beta$. More precisely, if $\alpha=0$ we can impose $\tilde{R}(a, b) \in\{0, \beta\}$ when $R(a, b)<\beta$.

Proof. Define the auxiliary relation $R_{0}$ as follows:

$$
R_{0}(a, b)= \begin{cases}0 & \text { if } R(a, b)=0 \\ 1 & \text { otherwise }\end{cases}
$$

This relation is crisp, reflexive, and transitive. Furthermore it is a compatible extension of $R$. For one thing, $R_{0} \geq R$ is trivial. For another, in order to prove $P_{0} \geq P$, where $P_{0}$ is the asymmetric part of $R_{0}$, observe that $P(a, b)>0$ is equivalent to $R(a, b)>0$ and $R(b, a)=0$. Therefore $P(a, b)>0$ is equivalent to the conjunction of $R_{0}(a, b)=1$ and $R_{0}(b, a)=0$, hence $P_{0}(a, b)=1$ follows.

We distinguish two cases depending on the value of $\alpha$.

Firstly we argue under the assumption $\alpha=0$. Then there exists a (crisp) total preorder $\tilde{R}_{0}$ that is a compatible extension of $R_{0}$ such that $\tilde{P}_{0}\left(b_{0}, a_{0}\right)=1$ (cf., Alcantud [1]). Now define the fuzzy relation $\tilde{R}$ as follows:

$$
\tilde{R}(a, b)= \begin{cases}R(a, b) & \text { if } R(a, b) \geq \beta \\ \beta & \text { if } R(a, b)<\beta \wedge \tilde{P}_{0}(b, a)=0 \\ 0 & \text { if } \tilde{P}_{0}(b, a)>0(\text { which implies } R(a, b)=0) .\end{cases}
$$

It is clear that $\tilde{R}(a, b)=R(a, b)$ for every $a, b$ such that $R(a, b) \geq \beta$. Also, $\tilde{R}\left(a_{0}, b_{0}\right)=R\left(a_{0}, b_{0}\right)=0$ because $\tilde{P}_{0}\left(b_{0}, a_{0}\right)=1$.

Also, by definition $\tilde{R}(a, b) \in\{0, \beta\}$ when $R(a, b)<\beta$.

We claim that $\tilde{R}$ is a compatible extension of $R$ and that $\tilde{R}$ is a total preorder.

Let us first see that $\tilde{R}$ is a compatible extension of $R$. The fact $\tilde{R}(a, b) \geq$ $R(a, b)$ for all $a, b \in X$ follows from the definition of $\tilde{R}$. In order to check $\tilde{P}(a, b) \geq P(a, b)$ for all $a, b \in X$, we can assume that $a, b$ verify $P(a, b)>0$ to avoid trivialities. In this case $R(a, b)>0$ and $R(b, a)=0$. Then $R_{0}(a, b)=1$ and $R_{0}(b, a)=0$, so that $P_{0}(a, b)=1$ and since $\tilde{R}_{0}$ is a compatible extension of $R_{0}$, we obtain $\tilde{P}_{0}(a, b)=1$. From this fact we deduce $\tilde{R}(b, a)=0$. Therefore $\tilde{P}(a, b)=\tilde{R}(a, b) \geq R(a, b) \geq P(a, b)$. 
We now check that $\operatorname{Trans}(R) \leq \operatorname{Trans}(\tilde{R})$. Fix any three alternatives $a, b, c \in X$. We will prove that $R(a, b) \wedge R(b, c) \rightarrow R(a, c) \leq \tilde{R}(a, b) \wedge \tilde{R}(b, c) \rightarrow$ $\tilde{R}(a, c)$.

First assume that $R(a, b) \wedge R(b, c) \rightarrow R(a, c)=R(a, c)$. Since $R \subseteq \tilde{R}$,

$$
R(a, b) \wedge R(b, c) \rightarrow R(a, c)=R(a, c) \leq \tilde{R}(a, c) \leq(\tilde{R}(a, b) \wedge \tilde{R}(b, c) \rightarrow \tilde{R}(a, c)) .
$$

Now assume that $R(a, b) \wedge R(b, c) \rightarrow R(a, c)=1$, this is, that $R(a, b) \wedge$ $R(b, c) \leq R(a, c)$. We have to prove that $\tilde{R}(a, b) \wedge \tilde{R}(b, c) \leq \tilde{R}(a, c)$. In order to prove $\tilde{R}(a, c) \geq \min (\tilde{R}(a, b), \tilde{R}(b, c))$ we distinguish three cases.

Case 1: $\tilde{R}(a, c)>\beta$. Thus $\tilde{R}(a, c)=R(a, c)$. If either $\tilde{R}(a, b) \leq \beta$ or $\tilde{R}(b, c) \leq \beta$ then the inequality follows trivially. Otherwise $\tilde{R}(a, b)>\beta$ and $\tilde{R}(b, c)>\beta$ thus by definition $\tilde{R}(a, b)=R(a, b)$ and $\tilde{R}(b, c)=R(b, c)$. Since $R$ is min-transitive,

$$
\tilde{R}(a, c)=R(a, c) \geq \min (R(a, b), R(b, c))=\min (\tilde{R}(a, b), \tilde{R}(b, c)) .
$$

Case 2: $\tilde{R}(a, c)=\beta$. Then $R(a, c) \leq \beta$. By min-transitivity of $R$, either $R(a, b) \leq \beta$ or $R(b, c) \leq \beta$ or both hold true. This implies that either $\tilde{R}(a, b) \leq \beta$ or $\tilde{R}(b, c) \leq \beta$, and the inequality follows immediately.

Case 3: $\tilde{R}(a, c)<\beta$, which is equivalent to $\tilde{R}(a, c)=0$. This yields $R(a, c)=0$ and $\tilde{P}_{0}(c, a)>0$. Since $R$ is min-transitive, $R(a, c) \geq \min (R(a, b), R(b, c))$. We assume without loss of generality that $R(a, b)=0$. If $\tilde{R}(a, b)=0$ we are done. Assume $\tilde{R}(a, b)>0$. Since $R(a, b)=0$ one obtains $\tilde{P}_{0}(b, a)=0$. Now $\tilde{R}_{0}(a, b)=1$ because $\tilde{R}_{0}$ is complete. Then

$$
0=\tilde{R}_{0}(a, c) \geq \min \left(\tilde{R}_{0}(a, b), \tilde{R}_{0}(b, c)\right)=\tilde{R}_{0}(b, c)
$$

henceforth $\tilde{R}_{0}(b, c)=0$. From this equality completeness yields $\tilde{R}_{0}(c, b)=1$ thus $\tilde{P}_{0}(c, b)=1$ therefore $\tilde{R}(b, c)=0$.

It remains to prove that $\operatorname{Re} f(R) \leq \operatorname{Re} f(\tilde{R})$ and that $\tilde{R}$ is complete. Since $\tilde{R}$ extends $R$ it holds that $R(a, a) \leq \tilde{R}(a, a)$ for every $a \in X$ and then $\bigwedge_{a \in X} R(a, a) \leq$ $\bigwedge_{a \in X} \tilde{R}(a, a)$. To prove completeness let us assume that $a, b \in X$ are such that $\tilde{R}(a, b)=0$. We proceed to check $\tilde{R}(b, a)>0$.

The equality $\tilde{R}(a, b)=0$ is equivalent to $R(a, b)=0$ and $\tilde{P}_{0}(b, a)>0$. Then $\tilde{P}_{0}(a, b)=0$ because $\tilde{P}_{0}$ is asymmetric. This guarantees $\tilde{R}(b, a)>0$ by the definition of $\tilde{R}$.

Secondly we argue under the assumption $\alpha>0$. Then there exists a (crisp) total preorder $\tilde{R}_{0}$ that is a compatible extension of $R_{0}$ (see for example Arrow [3]). Take $\delta=\min \{\alpha, \beta\}$. One solution is given by $\tilde{R}$ defined by the expression

$$
\tilde{R}(a, b)= \begin{cases}R(a, b) & \text { if } R(a, b) \geq \delta \\ \delta & \text { if } R(a, b)<\delta \wedge \tilde{P}_{0}(b, a)=0, \\ 0 & \text { if } \left.\tilde{P}_{0}(b, a)>0 \text { (which implies } R(a, b)=0\right) .\end{cases}
$$

By mimicking the argument above in this proof one easily checks that $(i) \tilde{R}$ is a compatible extension of $R,($ ii $) \tilde{R}$ is complete and $\operatorname{Re} f(R) \leq \operatorname{Ref}(\tilde{R})$ and 
$\operatorname{Trans}(R) \leq \operatorname{Trans}(\tilde{R}),($ iii $) \tilde{R}(a, b)=R(a, b)$ for all $a, b$ such that $R(a, b) \geq \delta$, and $(i v) \tilde{R}(a, b) \leq \delta$ for all $a, b$ such that $R(a, b)<\delta$. In particular, $\tilde{R}\left(a_{0}, b_{0}\right)=$ $R\left(a_{0}, b_{0}\right)=\alpha \geq \delta$ and $\tilde{R}(a, b)=R(a, b)$ for all $a, b \in X$ with $R(a, b) \geq \beta$ because $\beta \geq \delta$. Moreover, $\tilde{R}(a, b) \leq \beta$ for all $a, b$ such that $R(a, b)<\beta$ because if $R(a, b)<\delta$ then $\tilde{R}(a, b) \leq \delta \leq \beta$, otherwise $\delta \leq R(a, b)<\beta$ and then $\tilde{R}(a, b)=R(a, b)<\beta$.

Broadly speaking, Proposition 5.1 says that when extending a fuzzy relation to a fuzzy total relation, one can impose the condition that the degree to which a given element is in relation with another one can always be kept. The statement provided by this proposition is very versatile. It is applied in Corollaries 5.1 to 5.3 below, which do not need to refer to indicators. The interested reader could use it to derive other related statements too.

Corollary 5.1. Given a fuzzy preorder $R$ defined on a set $X$, let $\left\{\left(a_{i}, b_{i}\right)\right\}_{i=1}^{n}$ be a finite list of pairs of elements in $X$. If one of the following assumptions holds true

a) $R\left(a_{i}, b_{i}\right)>0$ for all $i=1, \ldots, n$ or

b) $R\left(a_{1}, b_{1}\right)=0$ and $R\left(a_{i}, b_{i}\right)>0$ for all $i \geq 2$,

then there exists a fuzzy total preorder $\tilde{R}$ that is a compatible extension of $R$ and such that $\tilde{R}\left(a_{i}, b_{i}\right)=R\left(a_{i}, b_{i}\right)$ for all $i=1, \ldots, n$.

Proof. Suppose first $R\left(a_{i}, b_{i}\right)>0$. Take $\alpha=\beta=\min \left(R\left(a_{1}, b_{1}\right), \ldots, R\left(a_{n}, b_{n}\right)\right)>$ 0 . Proposition 5.1 assures that there exists a fuzzy total preorder $\tilde{R}$ that is a compatible extension of $R$ such that $\tilde{R}(x, y)=R(x, y)$ for all $x, y$ satisfying $R(x, y) \geq \beta$. In particular, $\tilde{R}\left(a_{i}, b_{i}\right)=R\left(a_{i}, b_{i}\right)$ for all $i=1, \ldots, n$.

Suppose now $R\left(a_{i}, b_{i}\right)=0$. Take $\beta=\min \left\{R\left(a_{2}, b_{2}\right), \ldots, R\left(a_{n}, b_{n}\right)\right\}$ and $\alpha=0$. By Proposition 5.1, there exists a total preorder $\tilde{R}$ that is a compatible extension of $R$ such that $\tilde{R}\left(a_{1}, b_{1}\right)=R\left(a_{1}, b_{1}\right)=\alpha$ and $\tilde{R}(a, b)=R(a, b)$ for all $R(a, b) \geq \beta$. In particular, $\tilde{R}\left(a_{i}, b_{i}\right)=R\left(a_{i}, b_{i}\right)$ for all $i \geq 2$.

Remark 5.1. It is easy to prove that the conclusion of Corollary 5.1 does not hold if more than one pair of alternatives are not connected by $R$ and we want to preserve such disconnections. Consider for example the preorder $R$ defined on $X=\{a, b, c, d\}$ by $R(a, b)=1, R(c, d)=1, R(x, x)=1$ for all $x \in X$, and $R(x, y)=0$ otherwise. We argue that it is not possible to obtain a total preorder $\tilde{R}$ that is a compatible extension of $R$ such that both $\tilde{R}(a, d)=0$ and $\tilde{R}(c, b)=0$ hold true. Using that $\tilde{R}$ should be total, because $\tilde{R}(a, d)=0$ then $\tilde{R}(d, a)>0$. By transitivity, $\tilde{R}(d, b) \geq \min (\tilde{R}(d, a), \tilde{R}(a, b))>0$ thus $\tilde{R}(c, b) \geq \min (\tilde{R}(c, d), \tilde{R}(d, b))>0$, a contradiction.

Corollary 5.2. Given a fuzzy preorder $R$ defined on a set $X$, let us fix $a_{0}, b_{0} \in$ $X$ such that $R\left(a_{0}, b_{0}\right)=\alpha$ and $\beta>0$. Then there exists a fuzzy total preorder $\tilde{R}$ that is a compatible extension of $R$ and such that $\tilde{R}\left(a_{0}, b_{0}\right)=R\left(a_{0}, b_{0}\right)$ and $\tilde{R}(a, b)=R(a, b)$ for all $a, b$ with $R(a, b) \geq \beta$.

Furthermore, we can impose $\tilde{R}(a, b) \leq \beta$ when $R(a, b)<\beta$. More precisely, if $\alpha=0$ we can impose $\tilde{R}(a, b) \in\{0, \beta\}$ when $R(a, b)<\beta$. 
Corollary 5.3. Given a fuzzy preorder $R$ defined on a set $X$, let $\left\{\left(a_{i}, b_{i}\right)\right\}_{i=1}^{n}$ be a finite list of pairs of elements in $X$ such that $I\left(a_{i}, b_{i}\right)>0$ for all $i=1, \ldots, n$. Then there exists a fuzzy total preorder $\tilde{R}$ that is a compatible extension of $R$ and such that $\tilde{I}\left(a_{i}, b_{i}\right)=I\left(a_{i}, b_{i}\right)$ for all $i=1, \ldots, n$.

Proof. Because $\beta=\min \left(I\left(a_{1}, b_{1}\right), \ldots, I\left(a_{n}, b_{n}\right)\right)=\min \left(R\left(a_{1}, b_{1}\right), R\left(b_{1}, a_{1}\right), \ldots\right.$, $\left.R\left(a_{n}, b_{n}\right), R\left(b_{n}, a_{n}\right)\right)>0$, Corollary 5.1 assures that there is a fuzzy total preorder $\tilde{R}$ that is a compatible extension of $R$ with $\tilde{R}\left(a_{i}, b_{i}\right)=R\left(a_{i}, b_{i}\right)$ and $\tilde{R}\left(b_{i}, a_{i}\right)=R\left(b_{i}, a_{i}\right)$ for all $i=1, \ldots, n$. Therefore for all $i=1, \ldots, n$ we obtain

$$
\tilde{I}\left(a_{i}, b_{i}\right)=\min \left(\tilde{R}\left(a_{i}, b_{i}\right), \tilde{R}\left(b_{i}, a_{i}\right)\right)=\min \left(R\left(a_{i}, b_{i}\right), R\left(b_{i}, a_{i}\right)\right)=I\left(a_{i}, b_{i}\right) .
$$

Proposition 5.1 is a useful tool for analysis too, and in Section 5.2 we benefit from it in order to prove a general representation result for fuzzy relations from which one can deduce that fuzzy preorders can be realized as an intersection of fuzzy total preorders.

\subsection{A general representation result for fuzzy relations}

In this subsection we complement the information that fuzzy preorders can be extended to fuzzy total preorders.

We aim at showing that in fact, fuzzy total preordering extensions and compatible fuzzy total preordering extensions provide respective representations of fuzzy preorders.

In fact the introduction of indicators permits to prove a general result about the representation of fuzzy relations, namely, Theorem 5.1 below, from which the aforementioned lucid statement is a direct consequence:

Theorem 5.1. Consider the minimum t-norm. Then every fuzzy relation $R$ is the intersection of the collection of fuzzy total relations $\tilde{R}$ that are mincompatible extensions (resp., compatible extensions) of it and satisfy $\operatorname{Re} f(R) \leq$ $\operatorname{Ref}(\tilde{R}), \operatorname{Trans}(R) \leq \operatorname{Trans}(\tilde{R})$.

Proof. We proceed to prove the first statement, from which the second one derives easily.

Let $R$ be a fuzzy relation. It suffices to prove that for any $a_{0}, b_{0} \in X$ either there is a min-compatible extension $\tilde{R}$ satisfying $\operatorname{Ref}(R) \leq \operatorname{Ref}(\tilde{R})$ and $\operatorname{Trans}(R) \leq \operatorname{Trans}(\tilde{R})$ such that $\tilde{R}\left(a_{0}, b_{0}\right)=R\left(a_{0}, b_{0}\right)$ or there exists a set $\left\{\hat{R}_{n}\right\}_{n \in \mathbb{N}}$ of min-compatible extensions of $R$ satisfying $\operatorname{Ref}(R) \leq \operatorname{Ref}(\hat{R})$ and $\operatorname{Trans}(R) \leq \operatorname{Trans}(\hat{R})$ and provide the equality $\left(\cap_{n \in \mathbb{N}} \hat{R}_{n}\right)\left(a_{0}, b_{0}\right)=R\left(a_{0}, b_{0}\right)$.

Case 1. The pair $\left(a_{0}, b_{0}\right)$ does not verify $R\left(a_{0}, b_{0}\right)=0=R\left(b_{0}, a_{0}\right)$. Then we prove that there exists a complete relation $\tilde{R}$ that is a min-compatible extension of $R$ such that $\operatorname{Ref}(R) \leq \operatorname{Ref}(\tilde{R})$ and $\operatorname{Trans}(R) \leq \operatorname{Trans}(\tilde{R})$ and $\tilde{R}\left(a_{0}, b_{0}\right)=$ $R\left(a_{0}, b_{0}\right)$. 
Suppose $R\left(a_{0}, b_{0}\right)<R\left(b_{0}, a_{0}\right)$. Then for any $\bar{R}$ min-compatible extension it holds that $\bar{R}\left(a_{0}, b_{0}\right)=R\left(a_{0}, b_{0}\right)$ by the definition of min-compatible extension, because $\bar{R}(a, b) \leq R(b, a) \rightarrow R(a, b)=R(a, b)$ for all $a, b$.

Now assume $R\left(a_{0}, b_{0}\right) \geq R\left(b_{0}, a_{0}\right)$.

Select a total relation $\bar{R}$ that is a min-compatible extension of $R$ such that $\operatorname{Re} f(R) \leq \operatorname{Ref}(\bar{R})$ and $\operatorname{Trans}(R) \leq \operatorname{Trans}(\bar{R})$. Let $\beta=R\left(a_{0}, b_{0}\right)>0$ and consider the set $\left\{\left(a_{i}, b_{i}\right)\right\}_{i \in I}$ of all pairs of alternatives in $X$ such that $R\left(a_{i}, b_{i}\right)=0=R\left(b_{i}, a_{i}\right)$ and $\bar{R}\left(a_{i}, b_{i}\right)>0$. Then according to Proposition 5.1, for each $i \in I$ there exists a fuzzy total relation $\tilde{R}_{i}$ that is a compatible extension of $R$ and such that $\operatorname{Re} f(R) \leq \operatorname{Ref}(\bar{R}), \operatorname{Trans}(R) \leq \operatorname{Trans}(\bar{R})$ and $\tilde{R}_{i}\left(b_{i}, a_{i}\right)=0, \tilde{R}_{i}\left(a_{i}, b_{i}\right) \in\{0, \beta\}$ because $R\left(a_{i}, b_{i}\right)<\beta$, and $\tilde{R}_{i}\left(a^{\prime}, b^{\prime}\right)=R\left(a^{\prime}, b^{\prime}\right)$ whenever $R\left(a^{\prime}, b^{\prime}\right) \geqslant \beta$. In particular, $\tilde{R}_{i}\left(a_{0}, b_{0}\right)=\beta$ throughout.

Now consider $\tilde{R}=\left(\cup_{i \in I} \tilde{R}_{i}\right) \cap \bar{R}$. This relation satisfies that $\tilde{R}\left(a_{0}, b_{0}\right)=R\left(a_{0}, b_{0}\right)$ since $\tilde{R}\left(a_{0}, b_{0}\right) \leq\left(\cup_{i \in I} \tilde{R}_{i}\right)\left(a_{0}, b_{0}\right)=R\left(a_{0}, b_{0}\right)$ and $\bar{R}$ and all $\tilde{R}_{i}$ 's are extensions of $R$.

Let us check that $\tilde{R}$ is a min-compatible extension of $R$. It is clear that $R \subseteq \tilde{R}$ because $R \subseteq \bar{R}$ and $R \subseteq \tilde{R}_{i}$ for all $i \in I$. Since $\bar{R}$ is a min-compatible extension of $R$, for all $(a, b)$ such that $R(a, b)<R(b, a)$ it must be the case that $\bar{R}(a, b)=R(a, b)$. We deduce $\tilde{R}(a, b)=R(a, b)$ by using the fact that $\tilde{R}$ is an extension of $R$.

We now check that $\tilde{R}$ is total. For every $(a, b)$ such that $R(a, b)=0=R(b, a)$, either $\bar{R}(a, b)>0$ or $\bar{R}(b, a)>0$. Assuming without loss of generality that $\bar{R}(a, b)>0$, there must exist $i \in I$ such that $\tilde{R}_{i}(a, b)>0$. Then $\cup_{i \in I} \tilde{R}_{i}(a, b)>0$ and $\tilde{R}(a, b)=\left(\left(\cup_{i \in I} \tilde{R}_{i}\right) \cap \bar{R}\right)(a, b)>0$. So that $\tilde{R}$ is total.

Let us now prove that $\operatorname{Trans}(R) \leq \operatorname{Trans}(\tilde{R})$. Let us denote $\operatorname{Trans}(R)=\beta_{0}$. We will prove that for any $a, b, c \in X$ such that $\tilde{R}(a, b)<\beta_{0}$ it holds that $\tilde{R}(a, b) \geq \tilde{R}(a, c) \wedge \tilde{R}(c, b)$. Observe that since $\operatorname{Trans}(\bar{R}) \geq \operatorname{Trans}(R)$, if $\tilde{R}(a, b)=\bar{R}(a, b)$, then $\tilde{R}(a, b)=\bar{R}(a, b) \geq \min (\bar{R}(a, c), \bar{R}(c, b)) \geq \min (\tilde{R}(a, c), \tilde{R}(c, b))$ then we conclude. We will then assume that $\tilde{R}(a, b)=\cup_{i \in I} \tilde{R}_{i}(a, b)<\bar{R}(a, b)$. Assume firstly that $\tilde{R}(a, b)<\beta$. Because $\tilde{R}_{i}(a, b) \in\{0, \beta\}$ for all $i \in I$, the only possibility is $\tilde{R}_{i}(a, b)=0$ for all $i \in I$. This assumption immediately yields $\bar{R}(a, b)=0$. But we are assuming $\tilde{R}(a, b)=\cup_{i \in I} \tilde{R}_{i}(a, b)<\bar{R}(a, b)$.

Now assume $\beta_{0}>\tilde{R}(a, b)=\cup_{i \in I} \tilde{R}_{i}(a, b) \geq \beta$. Then $\beta_{0}>\tilde{R}_{i}(a, b)=R(a, b)$ for all $i$ by the construction of each $\tilde{R}_{i}$. Remember that since $\operatorname{Trans}(R)=\beta_{0}$, $R(a, b) \geq \min (R(a, c), R(c, b))$. Assume without loss of generality $R(a, b) \geq$ $R(a, c)$, the case $R(a, b) \geq R(c, b)$ being analogous. If $R(a, c) \geq \beta$, then $\tilde{R}(a, c)=\cup_{i \in I} \tilde{R}_{i}(a, c)=R(a, c)$ and we conclude. If $R(a, c)<\beta$, then $\tilde{R}_{i}(a, c) \leq$ $\beta$ for all $i \in I$, then it follows that $\tilde{R}(a, c) \leq \cup_{i \in I} \tilde{R}_{i}(a, b) \leq \beta$, whereas $\tilde{R}(a, b) \geq \beta \geq \tilde{R}(a, c)$.

It remains to prove that $\operatorname{Re} f(R) \leq \operatorname{Re} f(\tilde{R})$. Since $\tilde{R}$ extends $R$ it holds that $R(a, a) \leq \tilde{R}(a, a)$ for every $a \in X$ and then $\bigwedge_{a \in X} R(a, a) \leq \bigwedge_{a \in X} \tilde{R}(a, a)$.

Therefore $\tilde{R}$ is a total relation that is a min-compatible extension of $R$ and such that $\operatorname{Re} f(R) \leq \operatorname{Ref}(\tilde{R}), \operatorname{Trans}(R) \leq \operatorname{Trans}(\tilde{R})$ and $\tilde{R}\left(a_{0}, b_{0}\right)=R\left(a_{0}, b_{0}\right)$.

Case 2. The pair $\left(a_{0}, b_{0}\right)$ verifies $R\left(a_{0}, b_{0}\right)=0=R\left(b_{0}, a_{0}\right)$.

If there is a total relation $\bar{R}$ that is a min-compatible extension of $R$ such that 
$\operatorname{Re} f(R) \leq \operatorname{Re} f(\tilde{R}), \operatorname{Trans}(R) \leq \operatorname{Trans}(\tilde{R})$ and $\bar{R}\left(a_{0}, b_{0}\right)=0$ then we are done. Otherwise we select a total relation $\bar{R}$ that is a min-compatible extension of $R$ such that $\operatorname{Re} f(R) \leq \operatorname{Re} f(\tilde{R}), \operatorname{Trans}(R) \leq \operatorname{Trans}(\tilde{R})$ and verifies $\bar{R}\left(a_{0}, b_{0}\right)>0$.

Now consider $\beta=1 / n$ for $n$ a natural number and the set $\left\{\left(a_{i}, b_{i}\right)\right\}_{i \in I}$ of all pairs of alternatives in $X$ such that $R\left(a_{i}, b_{i}\right)=0=R\left(b_{i}, a_{i}\right)$ and $\bar{R}\left(a_{i}, b_{i}\right)>0$. Then according to Proposition 5.1, for each $\left(b_{i}, a_{i}\right)$ there exists a total relation $\tilde{R}_{i}$ that is a compatible extension of $R$ and such that $\operatorname{Re} f(R) \leq \operatorname{Re} f(\tilde{R})$, $\operatorname{Trans}(R) \leq \operatorname{Trans}(\tilde{R}) \tilde{R}\left(b_{i}, a_{i}\right)=0, \tilde{R}\left(a_{i}, b_{i}\right) \in\{0,1 / n\}$ because $R\left(a_{i}, b_{i}\right)<$ $1 / n$, and $\tilde{R}_{i}\left(a^{\prime}, b^{\prime}\right)=R\left(a^{\prime}, b^{\prime}\right)$ whenever $R\left(a^{\prime}, b^{\prime}\right) \geq 1 / n$. Moreover, $\tilde{R}_{i}\left(a_{0}, b_{0}\right) \in$ $\{0,1 / n\}$ because $R\left(a_{0}, b_{0}\right)=0<1 / n$. Denote $\hat{R}_{n}=\left(\cup_{i \in I} \tilde{R}_{i}(a, b)\right) \cap \bar{R}$. By mimicking the argument above in this proof, one easily checks that the relation $\hat{R}_{n}$ is a total relation that is a min-compatible extension of $R$ such that $\operatorname{Re} f(R) \leq$ $\operatorname{Re} f(\tilde{R}), \operatorname{Trans}(R) \leq \operatorname{Trans}(\tilde{R})$. Moreover, since $\tilde{R}_{i}\left(a_{0}, b_{0}\right) \leq \beta=1 / n$ for all $i \in I$, then $\hat{R}_{n}\left(a_{0}, b_{0}\right) \leq 1 / n$. Then for each $n$ we have that $\hat{R}_{n}$ is a relation that is a min-compatible extension of $R$ such that $\operatorname{Re} f(R) \leq \operatorname{Ref}(\tilde{R}), \operatorname{Trans}(R) \leq$ $\operatorname{Trans}(\tilde{R})$ satisfying $\hat{R}_{n}\left(a_{0}, b_{0}\right) \leq 1 / n$. Therefore $\cap_{n \in \mathbb{N}} \hat{R}_{n}\left(a_{0}, b_{0}\right)=0=R\left(a_{0}, b_{0}\right)$.

Theorem 5.1 can be regarded as a (double) fuzzy generalization of Donaldson and Weymark's [15] intersection theorem, thus answering an open question in Georgescu [17, Sect. 1]. It bears some comparison with Höhle and Blanchard [25, Corollary II.8], where linear order extensions of antisymmetric preorders are considered instead. And it introduces indicators into the analysis of the representability of fuzzy relations.

We can now derive a clear-cut statement on the representation of fuzzy preorders as follows:

Corollary 5.4. Consider the minimum t-norm. Then every fuzzy preorder $R$ is the intersection of the collection of fuzzy total preorders $\tilde{R}$ that are mincompatible extensions (resp., compatible extensions) of it.

\section{Concluding remarks}

In this contribution we continue the line of inspection in Alcantud and Díaz [2]. The driving question of our analysis is: to what extent can the characterizations presented by Alcantud [1] be generalized to the fuzzy set context? The main contribution of [1] is the proposal of necessary and sufficient conditions to ensure the existence of a crisp total preorder extension of a crisp preorder when some additional conditions are imposed, which extends the approach in the Szpilrajn theorem. For the analysis of the fuzzy counterpart of the problem, the main concept under inspection -compatible extensions- depends on the construction of associated strict preference relations. In our companion paper [2] we consider 
the widely acknowledged construction for the strict preference and the indifference relations based on generators. In this paper we focus on the alternative construction used by Georgescu [17].

Our results prove that the tighter construction adopted here is more suitable to produce arguments on compatible extensions of fuzzy preorders. We believe that this fact supports the view that this alternative construction is much closer to the crisp spirit than the construction from generators.

Furthermore, the fact that some results are related to the indicators of the discussed properties means that they can be expressed in a fuzzy logic and thanks to Georgescu [21, Section 3], integrated into fuzzy modal systems.

\section{Acknowledgements}

Both authors acknowledge financial support by the Spanish Ministerio de Economía y Competitividad, the first one under Project ECO2015-66797-P, the second one under Project TIN2014-59543-P.

\section{References}

[1] J. C. R. Alcantud. Conditional ordering extensions. Economic Theory, 39, 495-503 (2009).

[2] J. C. R. Alcantud, S. Díaz. Conditional extensions of fuzzy preorders. Fuzzy Sets and Systems, 278, 3-19 (2015).

[3] K. J. Arrow. Social Choice and Individual Values, 2nd ed. John Wiley \& Sons, 1963.

[4] R. Belohlavek, Fuzzy Relational Systems: Foundations and Principles. Kluwer Academic/Plenum Press (Vol. 20 of IFSR International Series on Systems Science and Engineering), New York, 2002.

[5] U. Bodenhofer, F. Klawonn. A formal study of linearity axioms for fuzzy orderings. Fuzzy Sets and Systems, 145, 323-354 (2004).

[6] G. Bosi, G. Herden. On a strong continuous analogue of the Szpilrajn theorem and its strengthening by Dushnik and Miller. Order, 22, 329-342 (2005).

[7] G. Bosi, G. Herden. On a possible continuous analogue of the Szpilrajn theorem and its strengthening by Dushnik and Miller. Order, 23, 271-296 (2006).

[8] W. Bossert. Intersection quasi-orderings: An alternative proof. Order, 16, 221-225 (1999).

[9] W. Bossert, Y. Sprumont, K. Suzumura. Upper semicontinuous extensions of binary relations. Journal of Mathematical Economics, 37, 231-246 (2002). 
[10] M. Dasgupta, R. Deb. Factoring fuzzy transitivity, Fuzzy Sets and Systems, 118, 489-502 (2001).

[11] De Baets, B., Fodor, J.: Additive fuzzy preference structures: the next generation. In: De Baets B., Fodor, J. (eds.) Principles of Fuzzy Preference Modelling and Decision Making, pp. 15-25. Academia Press (2003)

[12] B. De Baets, J. Fodor. Twenty years of fuzzy preference structures (19781997). JORBEL, 37, 61-82 (1997).

[13] S. Díaz, B. De Baets, S. Montes. On the compositional characterization of complete fuzzy pre-orders, Fuzzy Sets and Systems, 159, 2221-2239 (2008).

[14] S. Díaz, B. De Baets, S. Montes. General results on the decomposition of transitive fuzzy relations, Fuzzy Optimization and Decision Making, 9, 1-29 (2010).

[15] D. Donaldson and J. A. Weymark. A quasiordering is the intersection of orderings. Journal of Economic Theory, 78, 382-387 (1998).

[16] B. Dushnik and E. W. Miller. Partially ordered sets. American Journal of Mathematics, 63, 600-610 (1941).

[17] I. Georgescu. Compatible extensions of fuzzy relations. Journal of Systems Science and Systems Engineering, 12, 332-349 (2003)..

[18] I. Georgescu. Fuzzy Choice Functions: A Revealed Preference Approach. Springer, 2007.

[19] I. Georgescu. Compatible Extensions and Consistent Rationalizability: A Fuzzy Approach. TUCS Technical Reports 881, Turku Centre for Computer Science, 2008.

[20] I. Georgescu. Fuzzy Szpilrajn theorem through indicators. International Journal of Computers, Communications and Control, III (4), 336-343 (2008).

[21] I. Georgescu. On indicators of fuzzy relations. Annals of Fuzzy Mathematics and Informatics, 9 (4), 553-571 (2015).

[22] S. Gottwald. Fuzzy Sets and Fuzzy Logic. Vieweg, 1993.

[23] B. Hansson, Choice structures and preference relations. Synthese, 18, 443458 (1968).

[24] G. Herden, A. Pallack. On the continuous analogue of the Szpilrajn Theorem I. Mathematical Social Sciences, 43, 115-134 (2002).

[25] U. Höhle, N. Blanchard. Partial ordering in L-underdeterminate sets. Information Sciences, 35, 133-144 (1985). 
[26] J.-Y. Jaffray. Semicontinuous extension of a partial order. Journal of Mathematical Economics, 2, 395-406 (1975).

[27] E. Szpilrajn. Sur l'éxtension de l'ordre partiel. Fundamenta Mathematicae, 16, 386-389 (1930).

[28] G. Yi. Continuous extension of preferences. Journal of Mathematical Economics, 22, 547-555 (1993).

[29] L. Zadeh. Fuzzy sets. Information and Control, 3, 338-353 (1965).

[30] L. Zadeh. Similarity relations and fuzzy orderings. Information Sciences, 3, 177-200 (1971). 